\title{
Finanzierung der Zivilgesellschaft
}

\author{
Jana Priemer Ziviz | Bianca Corcoran-Schliemann Gfk | \\ Daniela Geue Deutscher Spendenrat | Holger Krimmer Ziviz | \\ Karsten Schulz-Sandhof DzI
}

\subsection{Konzeptionelle Einordnung ${ }^{1}$}

Engagierte Arbeit und Geld sind die beiden zentralen Ressourcen zivilgesellschaftlicher Organisationen. Der Faktor Arbeit lässt sich - wie in den vorangegangenen Kapiteln vorgenommen - differenzieren in bezahlte und unbezahlte, also ehrenamtlich geleistete, Arbeit. Der Faktor Geld lässt sich analog nach unterschiedlichen Finanzierungsquellen in vier Hauptgruppen differenzieren: Mitgliedsbeiträge, Spenden, selbsterwirtschaftete Mittel und Zuwendungen sowie Förderungen aus öffentlichen Quellen(vgl. Darstellung im Kasten, ZiviZ-Survey 2017, Kapitel 6.2). Letztere können insbesondere nach den föderalen Ebenen Kommune, Land und Bund weiter differenziert werden. Aber auch Mittel von der europäischen Ebene, unter anderem aus dem Europäischen Sozialfonds (ESF), und von anderen internationalen Akteuren müssen berücksichtigt werden.

Damit ergibt sich ein komplexes Bild der Finanzierung zivilgesellschaftlichen Handelns, das häufig auch auf Ebene einzelner Organisationen ähnlich komplex bleibt. Denn zahlreiche Vereine, Stiftungen, gGmbHs und andere Organisationen kombinieren in ihrem jeweiligen Finanzierungsmix Einnahmen aus ganz unterschiedlichen Tätigkeiten und Quellen. Ein gutes Verständnis der hybriden Finanzierungslogik ist gleichermaßen eine Voraussetzung für das Verständnis der Arbeitsweise und Rahmenbedingungen wie auch der Anforderungen an das Management zivilgesellschaftlicher Organisationen.

Häufig ist die Rede davon, dass Zivilgesellschaft „notorisch unterfinanziert“ sei. Tatsächlich müssen mit Blick auf die Finanzierung unterschiedliche Bereiche von Zivilgesellschaft voneinander differenziert werden. Wie in Kapitel 3 dargestellt, muss zunächst der sozialstaatsnahe Bereich, dem die Felder Gesund-

1 Dieses Unterkapitel wurde verfasst von Holger Krimmer, ZiviZ. 
heit, Soziale Dienste und teils auch die Bildung zuzurechnen sind, vom lokalen, mitgliedschaftsbasierten Vereinswesen der Bereiche Sport, Kultur und Freizeit/ Geselligkeit unterschieden werden. Auf der einen Seite stehen dienstleistungserbringende Organisationen mit einem hohen Grad an Verberuflichung ihrer Organisationsstrukturen, die ihre Arbeit über Leistungsentgelte von Kassen und Sozialversicherungsträgern refinanzieren können. Auf der anderen Seite finden sich überwiegend kleine Vereine ohne Beschäftigte, die zum größten Teil von Mitgliedsbeiträgen leben. Weitere Organisationstypen sind Organisationen, bei denen Spenden eine wichtige Rolle spielen, sowie Stiftungen, die sich typischerweise durch Vermögenserträge finanzieren. Stiftungen sind Vermögensmassen mit oder ohne eigene Rechtspersönlichkeit und zumindest im Idealfall von kontinuierlichen Refinanzierungszwängen befreit. Anzuführen sind auch die in den letzten Jahren laut ZiviZ-Survey 2017 zahlenmäßig stark wachsenden Fördervereine, deren Hauptzweck in der Regel darin besteht, finanzielle Ressourcen für ihre jeweiligen Mutterorganisationen zu mobilisieren.

Im Folgenden wird ein knapper Überblick über diese unterschiedlichen Finanzierungsarten und -modelle von Organisationen gegeben, bevor die Spendentätigkeit in Deutschland und die Finanzstruktur von Spendenorganisationen vertiefend diskutiert werden.

\subsection{Finanzierungsmix ${ }^{2}$}

Einnahmenhöhe: Die skizzierten Unterschiede zwischen den Organisationen zeigen sich besonders deutlich an den jährlichen Einnahmen. Die Spannweite reicht von kleinen, lokal aktiven Vereinen, die Einnahmen von weniger als 1 ooo Euro haben, bis hin zu national oder gar multinational agierenden Großorganisationen - einige mit hohen Beschäftigungsanteilen -, deren jährliche Einnahmen im mehrstelligen Millionenbereich liegen. Generell gilt jedoch: Die meisten Organisationen haben wenig Geld. Jede zweite Organisation hatte im Jahr 2015 maximal 10 ooo Euro zur Verfügung, ein Teil von ihnen noch viel weniger. Einnahmen in Millionenhöhe haben nur 4,5 Prozent der Organisationen. Besonders kleinen Vereinen steht meist nur wenig Geld zur Verfügung. Bei zwei von drei kleinen Vereinen (61,5 Prozent) mit bis zu 100 Mitgliedern lagen die Einnahmen im Jahr 2015 bei höchstens 10 ooo Euro. Hingegen hatten 85,4 Prozent der großen Vereine mit mehr als 300 Mitgliedern mehr als 10 ooo Euro zur Verfügung (vgl. Tab. 6.2). Herausragend hohe Einnahmen haben oftmals gemeinnützige GmbHs. Mehr als jede dritte gemeinnützige $\mathrm{GmbH}$ (41,8 Prozent) hat Einnahmen im Millionen-

2 Dieses Unterkapitel wurde verfasst von Jana Priemer, ZiviZ. 
Tabelle 6.1 Einnahmen (in Euro) im Jahr 2015 nach Rechtsform (in Prozent)

\begin{tabular}{|c|c|c|c|c|c|c|}
\hline & bis $10000 €$ & $\begin{array}{l}10001- \\
20000 €\end{array}$ & $\begin{array}{l}20001- \\
100000 €\end{array}$ & $\begin{array}{l}100001- \\
1 \text { Mio. } €\end{array}$ & $\begin{array}{l}\text { mehr als } \\
1 \text { Mio. } €\end{array}$ & Gesamt \\
\hline Verein & 51,0 & 12,1 & 20,6 & 12,5 & 3,8 & 100,0 \\
\hline Stiftung & 29,9 & 12,5 & 27,8 & 20,1 & 9,7 & 100,0 \\
\hline $\mathrm{gGmbH}$ & 13,9 & 2,5 & 7,6 & 34,2 & 41,8 & 100,0 \\
\hline Gesamt & 49,9 & 12 & 20,6 & 13 & 4,5 & 100,0 \\
\hline
\end{tabular}

Quelle: ZiviZ-Survey 2017, n = 6750 (gewichtet), davon fehlend: 1 004. Ohne Genossenschaften, da zu geringe Fallzahl.

Tabelle 6.2 Einnahmen (in Euro) im Jahr 2015 nach Vereinsgröße (in Prozent)

\begin{tabular}{|c|c|c|c|c|c|c|}
\hline & $\begin{array}{l}\text { bis } \\
10000 €\end{array}$ & $\begin{array}{l}10001- \\
20000 €\end{array}$ & $\begin{array}{l}20001- \\
100000 €\end{array}$ & $\begin{array}{l}100001- \\
1 \text { Mio. } €\end{array}$ & $\begin{array}{l}\text { mehr als } \\
1 \text { Mio. } €\end{array}$ & Gesamt \\
\hline klein (bis 100 Mitglieder) & 61,5 & 11,4 & 14,3 & 9,8 & 3,1 & 100 \\
\hline $\begin{array}{l}\text { mittelgroß (101 bis } \\
300 \text { Mitglieder) }\end{array}$ & 45,7 & 16,1 & 26,2 & 8,9 & 3,1 & 100 \\
\hline $\begin{array}{l}\text { groß (mehr als } 300 \text { Mit- } \\
\text { glieder) }\end{array}$ & 14,5 & 10,4 & 40,4 & 27,9 & 6,7 & 100 \\
\hline Gesamt & 50,8 & 12,4 & 21 & 12,3 & 3,6 & 100 \\
\hline
\end{tabular}

Quelle: ZiviZ-Survey 2017, nur Vereine, $\mathrm{n}=6461$ (gewichtet), davon fehlend: 1326.

bereich. Gemeinnützige GmbHs haben zwar in den vergangenen Jahren erheblich an Zahl zugenommen, dennoch sind sie nicht die vorherrschende Rechtsform der organisierten Zivilgesellschaft. Vielmehr handelt es sich um einen kleinen, sehr spezifisch aufgestellten Bereich (vgl. Kapitel 3), der sich hinsichtlich seiner Strukturmerkmale von Vereinen und Stiftungen unterscheidet. Dies zeigt sich in den Finanzierungsstrukturen besonders deutlich.

Zwischen den Handlungsfeldern zeigen sich ebenfalls erhebliche Unterschiede. Im Bevölkerungs- und Katastrophenschutz, zu dem vor allem Feuerwehrvereine, Mitgliedsvereine wie die Deutsche Lebens-Rettungs-Gesellschaft oder die Bergrettung sowie auch Fördervereine gehören, haben meist besonders kleine finanzielle Spielräume. Drei Viertel müssen mit weniger als 10 ooo Euro jährlich auskommen. Vereine mit Einnahmen in Millionenhöhe gibt es hier kaum. Ähnlich verhält es sich im Freizeitbereich: Auch unter den Freizeitvereinen gibt es 
nur wenige, die Einnahmen von mehr als einer Million Euro haben. Die meisten (69,1 Prozent) hatten im Jahr 2015 höchstens 10 ooo Euro. Ganz anders verhält es sich bei den Organisationen der Sozialen Dienste. Die Einnahmen der Organisationen, die sich in den Sozialen Diensten verorten, sind in den vergangenen Jahren überdurchschnittlich oft gestiegen. 60,1 Prozent geben an, dass ihre Einnahmen gegenüber dem Jahr 2008 gestiegen sind. Mit 19,9 Prozent gibt es heute die meisten Organisationen mit jährlichen Einnahmen von mehr als einer Million Euro in den Sozialen Diensten. Zu berücksichtigen ist bei dieser Entwicklung jedoch, dass es sich um ein sehr personalintensives Organisationssegment handelt, so dass - anders als in anderen Organisationsbereichen - ein Großteil der Einnahmen für Personalkosten verausgabt wird. Diese machen bei den Organisationen der Sozialen Dienste durchschnittlich 36,4 Prozent aus. Grundsätzlich gilt: Je höher die Gesamteinnahmen sind, desto höher ist auch der Anteil der Personalkosten. Bei den Einrichtungen der Sozialen Dienste mit jährlichen Einnahmen von mehr als einer Million Euro liegen sie bei durchschnittlich 69,o Prozent.

Hervorzuheben ist, dass es neben diesen zum Teil stark wirtschaftlich orientierten Organisationen auch im Handlungsfeld Soziale Dienste zahlreiche kleine, ehrenamtlich getragene Organisationen gibt, die nicht auf große finanzielle Ressourcen zurückgreifen können (Eckl 2015, S. 80 ff.). Mehr als jede vierte Organisation der Sozialen Dienste (28,1 Prozent) hat jährliche Einnahmen von höchstens 10000 Euro. Eine ähnlich große Bandbreite findet sich auch in anderen Handlungsfeldern, wie etwa im Bildungsbereich. Das Handlungsfeld Bildung besteht ebenfalls aus einem mit vergleichsweise guten finanziellen Ressourcen ausgestatteten Bereich hochprofessioneller Organisationen ${ }^{3}$ und aus einem rein bürgerschaftlich getragenen Bereich, der mit wenig Geld auskommen muss (Priemer und Mohr 2018; Priemer 2015).

Herkunft der Mittel: Für zivilgesellschaftliche Organisationen ist es zwar typisch, dass ihre Einnahmen aus verschiedenen Finanzierungsquellen kommen, doch bei den meisten Organisationen dominiert eine Finanzierungsquelle (Littich und Schober 2013, S. 289). Diese variieren, wie bereits angedeutet, je nach Organisationssegment zum Teil erheblich.

Mitgliedsbeiträge waren schon immer die wichtigste Einnahmequelle für Vereine (Horch 1992, S. 139 f.; Emrich et al. 2001, S. 216). Daran hat sich bis heute wenig geändert. Die finanziellen Ressourcen werden nach wie vor bei den meisten Ver-

3 Finanziell gut ausgestattet im Vergleich zu anderen Organisationen, nicht hinsichtlich ihrer Bedarfe. Mit hochprofessionell meinen wir, dass sie - anders als sonst im Dritten Sektor üblich - mit bezahlten Beschäftigten arbeiten. 
Tabelle 6.3 Einnahmen (in Euro) im Jahr 2015 kategorisiert nach Handlungsfeld (in Prozent)

\begin{tabular}{|c|c|c|c|c|c|c|}
\hline & $\begin{array}{l}\text { bis } \\
10000 €\end{array}$ & $\begin{array}{l}10001- \\
20000 €\end{array}$ & $\begin{array}{l}20001- \\
100000 €\end{array}$ & $\begin{array}{l}100001- \\
1 \text { Mio. } €\end{array}$ & $\begin{array}{l}\text { mehr als } \\
1 \text { Mio. } €\end{array}$ & Gesamt \\
\hline $\begin{array}{l}\text { Bevölkerungs-/Katastro- } \\
\text { phenschutz }\end{array}$ & 74,4 & 16,7 & 7,1 & 1,8 & 0,0 & 100 \\
\hline Freizeit/Geselligkeit & 69,1 & 10,0 & 19,0 & 1,9 & 0,0 & 100 \\
\hline Sonstiges & 64,5 & 16 & 6,9 & 10,8 & 1,7 & 100 \\
\hline Umwelt-/Naturschutz & 60,4 & 10,4 & 18,1 & 9,3 & 1,6 & 100 \\
\hline $\begin{array}{l}\text { Bürger-/Verbraucher- } \\
\text { interessen }\end{array}$ & 57,7 & 15,4 & 10,7 & 14,1 & 2 & 100 \\
\hline Kultur/Medien & 56,1 & 14,7 & 21,1 & 7,0 & 1,0 & 100 \\
\hline $\begin{array}{l}\text { Internationale Soli- } \\
\text { darität }\end{array}$ & 54 & 6,9 & 26,4 & 5,2 & 7,5 & 100 \\
\hline Gesamt & 49,8 & 12,0 & 20,7 & 13,0 & 4,5 & 100 \\
\hline $\begin{array}{l}\text { Kirchen/religiöse Ver- } \\
\text { einigungen }\end{array}$ & 49,1 & 8,5 & 24,1 & 16,5 & 1,8 & 100 \\
\hline $\begin{array}{l}\text { Wirtschafts-/Berufs- } \\
\text { verbände }\end{array}$ & 46,6 & 12,3 & 20,5 & 19,2 & 1,4 & 100 \\
\hline Wissenschaft/Forschung & 45,8 & 13,1 & 17,6 & 15,0 & 8,5 & 100 \\
\hline Bildung/Erziehung & 45,2 & 8,5 & 15,8 & 22,0 & 8,6 & 100 \\
\hline Sport & 43,8 & 14,3 & 28,7 & 11,9 & 1,3 & 100 \\
\hline Gesundheitswesen & 39,4 & 5,6 & 26,8 & 20,7 & 7,6 & 100 \\
\hline $\begin{array}{l}\text { Gemeinschaftliche } \\
\text { Versorgungsaufgaben* }\end{array}$ & 34 & 17 & 29,8 & 17,0 & 2,1 & 100 \\
\hline Soziale Dienste & 28,1 & 11,6 & 21,5 & 19,9 & 18,9 & 100 \\
\hline
\end{tabular}

Quelle: ZiviZ-Survey 2017, $\mathrm{n}=6750$ (gewichtet), davon fehlend: $1136,{ }^{*} \mathrm{n}<50$ (47). 


\section{Die im ZiviZ-Survey erfassten Einnahmequellen}

Mitgliedsbeiträge: „Klassische“ Beiträge für Mitgliedschaften in Vereinen, einschließlich Aufnahmegebühren. Fördermitgliedsbeiträge fallen in die Rubrik Spenden.

Erwirtschaftete Mittel (Markterträge): Jegliche Form von Leistungsentgelten und -pauschalen, auch aus öffentlichen Kassen, sowie Gebühren, Eintrittsgelder, Lotterieeinnahmen und ähnliches, einschließlich Mittel gesetzlicher Sozialversicherungen.

Spenden: Freiwillige Zahlungen von Privatpersonen, Stiftungen und Fonds, Unternehmen oder anderen Organisationen, einschließlich Fördermittel von Trägerorganisationen, Verbänden, Stiftungen, Fördermitgliedsbeiträge und ähnliches. Fördermitgliedschaften gibt es auch bei Rechtsformen, die normalerweise keine Mitgliedsbeiträge erheben, wie Stiftungen und gemeinnützige GmbHs.

Öffentliche Fördermittel: Institutionelle und projektbezogene Zuwendungen staatlicher Einrichtungen.

Sponsorengelder: Im Gegensatz zur Spende findet für die geleisteten Zahlungen ein Leistungstausch statt.

Vermögenserträge: Erträge aus Geldanlagen (Zinsen, Dividenden) sowie aus der Vermietung und Verpachtung von Grundbesitz und Immobilien (Mieten und Pachten).

Sonstige Mittel: Sondereinnahmen wie etwa Bußgelder, Erbschaften oder Rückbuchungen aus Vorjahren. 
einen über die Mitglieder gesichert. Im Durchschnitt machen Mitgliedsbeiträge bei den Vereinen 40,2 Prozent der Einnahmen aus. Bei großen Vereinen mit mehr als 300 Mitgliedern ist der Anteil mit 49,8 Prozent sogar noch etwas höher, bei kleinen Vereinen mit weniger als 100 Mitgliedern mit 36,2 Prozent hingegen geringer.

Für die anderen Rechtsformen spielen Mitgliedsbeiträge kaum eine Rolle. Stiftungen beispielsweise werden überwiegend über Vermögenserträge finanziert, die 2016 durchschnittlich 56,0 Prozent der Einnahmen ausmachen. Das ist geringfügig weniger als im Haushaltsjahr 2011 (6o,o Prozent). Die Spendenanteile hingegen sind minimal gestiegen. Der Anteil von Spenden an den Gesamteinnahmen der Stiftungen liegt heute bei 26,6 Prozent. Es ist zu vermuten, dass es sich hierbei um keine reale Steigerung der Spendenanteile handelt, sondern um eine relationale, da die Vermögenserträge aufgrund der andauernden Niedrigzinslage in den vergangenen Jahren gesunken sind. Tatsächlich berichtet jede dritte Stiftung (32,7 Prozent), dass die Einnahmen seit 2012 rückläufig sind. Bei den Vereinen sind es nur 19,7 Prozent und bei den gemeinnützigen GmbHs berichten sogar nur 10,3 Prozent von gesunkenen Einnahmen. Im Gegenteil: Bei 74,7 Prozent der gemeinnützigen GmbHs sind die Einnahmen gestiegen. Die vergleichsweise ausgeprägte wirtschaftliche Orientierung von gemeinnützigen GmbHs zeigt sich auch in ihren Finanzierungsquellen. Denn anders als Vereine oder Stiftungen sind sie mit einem durchschnittlichen Anteil von 48,5 Prozent zu erheblichen Teilen über wirtschaftliche Aktivitäten finanziert. Zudem spielen mit einem durchschnittlichen Anteil von 26,7 Prozent öffentliche Mittel für gemeinnützige GmbHs im Finanzierungsmix eine vergleichsweise große Rolle.

Mitgliedsbeiträge machen mit 79,1 Prozent bei den Wirtschafts- und Berufsverbänden einen besonders hohen Anteil am Finanzierungsmix aus. Auch im

Tabelle 6.4 Einnahmequellen 2017 nach Rechtsform (in Prozent)

\begin{tabular}{lcclllll}
\hline & $\begin{array}{l}\text { Mitglieds- } \\
\text { beiträge }\end{array}$ & $\begin{array}{l}\text { Erwirt- } \\
\text { schaftete } \\
\text { Mittel }\end{array}$ & Spenden & $\begin{array}{l}\text { Öffentliche } \\
\text { Förder- } \\
\text { mittel }\end{array}$ & $\begin{array}{l}\text { Sponso- } \\
\text { rengelder }\end{array}$ & $\begin{array}{l}\text { Vermögens- Sonstige } \\
\text { erträge }\end{array}$ & \\
\hline Verein & 40,2 & 19,9 & 18,7 & 10,9 & 4,1 & 1,8 & 2,2 \\
GmbH & 4,1 & 48,5 & 10,3 & 26,7 & 2,8 & 2,9 & 2,0 \\
Stiftung & 0,7 & 6,3 & 26,6 & 3,4 & 2,6 & 56,0 & 3,1 \\
\hline Gesamt & $\mathbf{3 8 , 6}$ & $\mathbf{2 0 , 1}$ & $\mathbf{1 8 , 8}$ & $\mathbf{1 1 , 0}$ & $\mathbf{4 , 1}$ & $\mathbf{3 , 3}$ & $\mathbf{2 , 2}$ \\
\hline
\end{tabular}

Quelle: ZiviZ-Survey 2017, n = 6750 (gewichtet), davon fehlend: 823, ohne Genossenschaften da zu geringe Fallzahl. 
Tabelle 6.5 Einnahmequellen 2017 nach Vereinsgröße (Mittelwerte der Prozentangaben)

\begin{tabular}{|c|c|c|c|c|c|c|c|}
\hline & $\begin{array}{l}\text { Mitglieds- } \\
\text { beiträge }\end{array}$ & $\begin{array}{l}\text { Erwirt- } \\
\text { schaftete } \\
\text { Mittel }\end{array}$ & Spenden & $\begin{array}{l}\text { Öffentliche } \\
\text { Förder- } \\
\text { mittel }\end{array}$ & $\begin{array}{l}\text { Sponso- } \\
\text { rengelder }\end{array}$ & $\begin{array}{l}\text { Vermögens- } \\
\text { erträge }\end{array}$ & Sonstige \\
\hline $\begin{array}{l}\text { klein (bis } \\
100 \text { Mitglie- } \\
\text { der) }\end{array}$ & 36,2 & 20,6 & 21,5 & 11,4 & 3,9 & 1,9 & 2,3 \\
\hline $\begin{array}{l}\text { mittelgroß } \\
\text { (101 bis } 300 \\
\text { Mitglieder) }\end{array}$ & 44,8 & 21,3 & 15,2 & 9,1 & 3,8 & 1,8 & 2,3 \\
\hline $\begin{array}{l}\text { groß (mehr } \\
\text { als } 300 \text { Mit- } \\
\text { glieder) }\end{array}$ & 49,8 & 17,7 & 11,6 & 10,3 & 6,3 & 2,2 & 1,1 \\
\hline
\end{tabular}

Quelle: ZiviZ-Survey 2017: nur Vereine, $\mathrm{n}=6461$ (gewichtet), davon fehlend: 1176.

Sport sind die Mitgliedsbeiträge, die hier durchschnittlich mehr als die Hälfte (56,0 Prozent) der Einnahmen ausmachen, ein zentraler Finanzierungsbestandteil. In anderen Handlungsfeldern spielen Mitgliedsbeiträge teilweise ebenfalls eine große Rolle, doch werden sie dort stärker durch andere Finanzierungsquellen ergänzt, bei den Organisationen der Internationalen Solidarität beispielsweise durch Spenden (vgl. Tabelle 6.5).

Erwirtschaftete Mittel: sind Markterträge. Sie spielen nicht nur für gemeinnützige GmbHs bei der Finanzierung eine wichtige Rolle, sondern auch für Vereine. Durch den Verkauf von Speisen und Getränken im Vereinstreff, den Verkauf von Eintrittskarten für kulturelle Veranstaltungen und andere Events oder die Bereitstellung anderer gebührenpflichtiger Angebote und Leistungen (zum Beispiel Seminare) finanzieren zahlreiche Vereine ihre Vereinsaktivitäten. Derartige Einnahmen machen bei Vereinen immerhin ein Fünftel (19,9 Prozent) der Einnahmen aus. Die Sozialen Dienste fallen durch besonders hohe Anteile von Markterträgen auf. Bei ihnen machen diese mit 28,0 Prozent mehr als ein Viertel der Gesamteinnahmen aus. Übertroffen werden sie nur von den Organisationen des Handlungsfelds Gemeinschaftliche Versorgungsaufgaben, bei denen erwirtschaftete Mittel mit 35,7 Prozent mehr als ein Drittel ausmachen. Dies liegt darin begründet, dass auf dieses Handlungsfeld besonders viele Genossenschaften (zum Beispiel Energiegenossenschaften, Konsumgenossenschaften und Sozialgenossenschaften) entfallen, die ähnlich wie gemeinnützige GmbHs einen stärkeren Wirtschaftsbezug haben als die meisten Vereine und Stiftungen. Bei der Finanzierung 
von Stiftungen spielen derartige Markterträge mit 6,3 Prozent kaum eine Rolle. Die bei ihnen dominierenden Vermögenserträge werden in einer separaten Kategorie erfasst (vgl. Tab. 6.5).

Spenden: sind nicht nur für Stiftungen eine wichtige Ergänzung im Finanzierungsmix. Die über Spenden generierten Finanzierungsanteile sind - bezogen auf alle Organisationen - mit 18,8 Prozent ähnlich hoch wie die, die durch wirtschaftliche Aktivitäten gewonnen werden. Vor allem für kleine Vereine mit wenigen Mitgliedern spielen Spenden offenbar eine wichtige Rolle. Bei Vereinen mit maximal 100 Mitgliedern liegt der über Spenden gewonnene Anteil der Einnahmen bei 21,5 Prozent, bei großen Vereinen mit mehr als 300 Mitgliedern hingegen nur bei 11,6 Prozent. Hierin spiegelt sich unter anderem der Trend zu Fördervereinen wider (vgl. Kapitel 3), die sich nicht nur über Fördermitgliedschaften finanzieren - Fördermitgliedsbeiträge entfallen in die Kategorie Spenden -, sondern auch über diverse Spendenaktivitäten. $\mathrm{Zu}$ den Spendenaktivitäten von Fördervereinen gehören etwa Spendenläufe/-basare oder Benefizveranstaltungen. Da es bei Fördervereinen nicht unüblich ist, dass sie von nur wenigen Mitgliedern getragen werden (Braun et al. 2013; Freise 2015), fällt der Einnahmeanteil durch Mitgliedsbeiträge bei ihnen oftmals relativ gering aus. Hinsichtlich der Handlungsfelder nehmen Spenden vor allem bei den Organisationen der Internationalen Solidarität (43,9 Prozent) und des Handlungsfeldes Kirchen und religiöse Vereinigungen (40,8 Prozent) eine zentrale Rolle ein.

Öffentliche Mittel: spielen im Finanzierungsmix zivilgesellschaftlicher Organisationen in der Realität eine geringere Rolle, als die Diskussion um die finanzielle Abhängigkeit der deutschen Zivilgesellschaft von staatlichen Mitteln (Anheier 1998; Priemer et al. 2016, S. 26 f.) vermuten ließe. Tatsächlich kommt nur ein kleiner Teil der Vereine und Stiftungen in den Genuss öffentlicher Mittel. So bezieht nur jeder dritte Verein (33,8 Prozent) öffentliche Fördermittel, von den Stiftungen sogar nur jede zehnte (11,1 Prozent). In hohem Maße über öffentliche Mittel finanziert sind hingegen die gemeinnützigen GmbHs. Diese sind vor allem in den Handlungsfeldern aktiv, in denen es besonders viele Organisationen und Einrichtungen gibt, die wohlfahrtsstaatliche Leistungen erbringen (Priemer et al. 2015, S. 26 f.). In diesen Handlungsfeldern gibt es nicht nur besonders viele Organisationen, die öffentliche Mittel beziehen, sondern dort sind auch die Anteile der öffentlichen Mittel am Gesamtfinanzierungsmix überdurchschnittlich hoch. Von den Organisationen der Sozialen Dienste beziehen 43,1 Prozent öffentliche Mittel. Dort machen die öffentlichen Mittel 18,1 Prozent an den Gesamteinnahmen aus. Werden nur jene Organisationen berücksichtigt, die überhaupt öffentliche Mittel beziehen, steigt der Anteil der Gesamteinnahmen durch öffentliche Mittel 
bei den Sozialen Diensten auf 42,o Prozent. Schaut man sich hingegen nur jene Organisationen an, die gar keine öffentlichen Mittel erhalten, dann finanzieren sich auch die Organisationen der Sozialen Dienste überwiegend über Spenden und Mitgliedsbeiträge. Diese machen dann einen Finanzierungsanteil von mehr als der Hälfte der Einnahmen aus (52,5 Prozent, davon 32,1 Prozent Spenden und 20,4 Prozent Mitgliedsbeiträge).

Eine ähnliche Situation lässt sich für das Handlungsfeld Bildung und Erziehung beschreiben. Hier beziehen 41,4 Prozent der Organisationen öffentliche Mittel. Am Gesamtfinanzierungsmix machen öffentliche Mittel 23,o Prozent aus. Auch hier steigen die Anteile der öffentlichen Mittel an, wenn nur jene Organisationen berücksichtigt werden, die überhaupt öffentliche Mittel beziehen. Der Anteil der öffentlichen Mittel steigt dann auf 55,5 Prozent. Bei der ausschließlichen Berücksichtigung jener Organisationen, die keine öffentlichen Mittel beziehen, bestimmen Mitgliedsbeiträge (37,2 Prozent) und Spenden (29,4 Prozent) zusammen zwei Drittel der Einnahmen.

Neben der Rechtsform hat auch die Organisationsgröße einen Einfluss auf den Bezug öffentlicher Mittel. Je größer die Organisation - in Bezug auf ihre Einnahmen und bei den Vereinen auf ihre Mitgliederzahlen -, desto wahrscheinlicher bezieht sie auch öffentliche Mittel. Kleine Organisationen hingegen bekommen nicht nur seltener öffentliche Mittel, sondern wenn sie welche bekommen, machen diese geringere Anteile am Gesamtfinanzierungsmix aus.

Abbildung 6.1 Vereine, die öffentliche Mittel erhalten, nach Vereinsgröße (in Prozent)

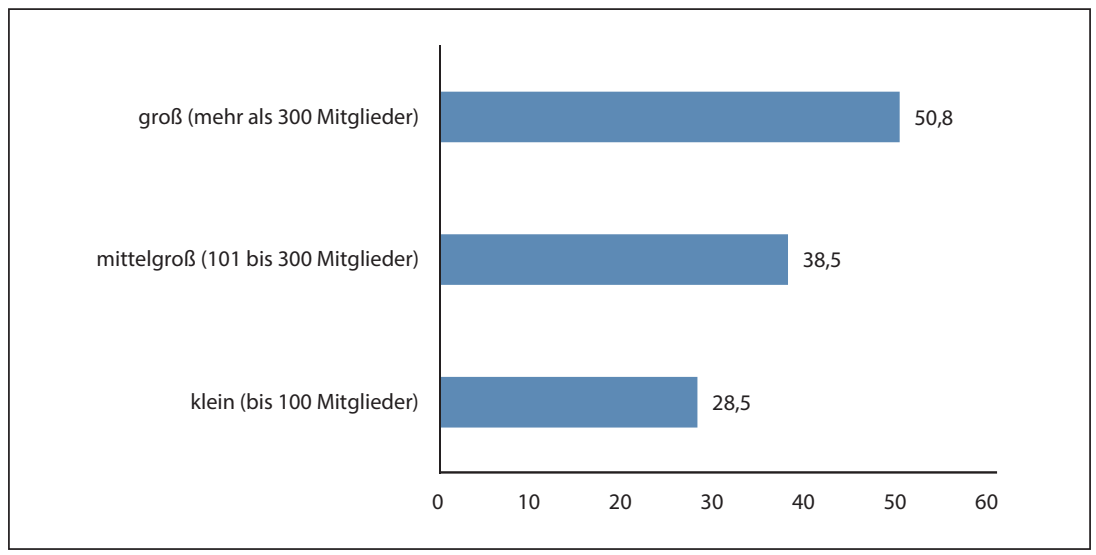

Quelle: ZiviZ-Survey 2017: nur Vereine, n = 6461 (gewichtet), davon fehlend: 1177. 
Tabelle 6.6 Einnahmequellen 2017 nach Handlungsfeld (Mittelwerte der Prozentangaben)

\begin{tabular}{|c|c|c|c|c|c|c|c|}
\hline & $\begin{array}{l}\text { Mitglieds- } \\
\text { beiträge }\end{array}$ & $\begin{array}{l}\text { Erwirt- } \\
\text { schaftete } \\
\text { Mittel }\end{array}$ & Spenden & $\begin{array}{l}\text { Öffentliche } \\
\text { Förder- } \\
\text { mittel }\end{array}$ & $\begin{array}{l}\text { Sponso- } \\
\text { rengelder }\end{array}$ & $\begin{array}{l}\text { Vermögens- } \\
\text { erträge }\end{array}$ & Sonstige \\
\hline $\begin{array}{l}\text { Wirtschafts-/ } \\
\text { Berufsver- } \\
\text { bände }\end{array}$ & 79,1 & 7,5 & 2,6 & 2,6 & 2,7 & 1,2 & 79,1 \\
\hline Sport & 56,0 & 16,1 & 11,1 & 5,7 & 5,6 & 2,1 & 56,0 \\
\hline $\begin{array}{l}\text { Freizeit/Ge- } \\
\text { selligkeit }\end{array}$ & 50,7 & 25,9 & 8,0 & 3,4 & 2,4 & 3,3 & 50,7 \\
\hline $\begin{array}{l}\text { Bürger-/ } \\
\text { Verbraucher- } \\
\text { interessen }\end{array}$ & 49,7 & 11,0 & 19,8 & 13,1 & 2,8 & 0,7 & 49,7 \\
\hline $\begin{array}{l}\text { Umwelt-/ } \\
\text { Naturschutz }\end{array}$ & 48,2 & 13,8 & 23,9 & 6,1 & 2,4 & 4,0 & 48,2 \\
\hline Sonstiges & 42,3 & 13,9 & 17,5 & 10,4 & 5,2 & 3,2 & 42,3 \\
\hline $\begin{array}{l}\text { Wissenschaft/ } \\
\text { Forschung }\end{array}$ & 40,8 & 10,0 & 15,2 & 10,4 & 3,3 & 12,0 & 40,8 \\
\hline Gesamt & 38,6 & 20,1 & 18,8 & 11,0 & 4,0 & 3,2 & 38,6 \\
\hline $\begin{array}{l}\text { Bevölke- } \\
\text { rungs-/ } \\
\text { Katastrophen- } \\
\text { schutz }\end{array}$ & 36,4 & 27,2 & 18,3 & 5,4 & 2,4 & 1,0 & 36,4 \\
\hline $\begin{array}{l}\text { Kultur/ } \\
\text { Medien }\end{array}$ & 34,5 & 28,0 & 17,2 & 9,0 & 5,1 & 2,7 & 34,5 \\
\hline $\begin{array}{l}\text { Gemein- } \\
\text { schaftliche } \\
\text { Versorgungs- } \\
\text { aufgaben* }\end{array}$ & 32,2 & 35,7 & 13,0 & 2,1 & 4,8 & 8,9 & 32,2 \\
\hline $\begin{array}{l}\text { Kirchen/reli- } \\
\text { giöse Vereini- } \\
\text { gungen }\end{array}$ & 30,1 & 12,3 & 40,8 & 3,7 & 1,0 & 5,1 & 30,1 \\
\hline $\begin{array}{l}\text { Gesundheits- } \\
\text { wesen }\end{array}$ & 26,7 & 21,6 & 27,6 & 12,4 & 4,8 & 3,2 & 26,7 \\
\hline $\begin{array}{l}\text { Bildung/ } \\
\text { Erziehung }\end{array}$ & 26,5 & 18,9 & 21,7 & 23,0 & 3,7 & 3,0 & 26,5 \\
\hline $\begin{array}{l}\text { Internationale } \\
\text { Solidarität }\end{array}$ & 20,8 & 16,2 & 43,9 & 9,6 & 2,0 & 3,2 & 20,8 \\
\hline $\begin{array}{l}\text { Soziale } \\
\text { Dienste }\end{array}$ & 16,0 & 28,0 & 25,1 & 18,1 & 3,2 & 5,1 & 16,0 \\
\hline
\end{tabular}

Quelle: ZiviZ-Survey 2017: alle Organisationen, $n=6750$ (gewichtet), davon fehlend: 963. 
Tabelle 6.7 Einnahmequellen (in Euro) 2017 nach Einnahmehöhe (Mittelwerte der Prozentangaben)

\begin{tabular}{lllllllr}
\hline & $\begin{array}{l}\text { Mitglieds- } \\
\text { beiträge }\end{array}$ & $\begin{array}{l}\text { Erwirt- } \\
\text { schaftete } \\
\text { Mittel }\end{array}$ & Spenden & $\begin{array}{l}\text { Öffentliche } \\
\text { Förder- } \\
\text { mittel }\end{array}$ & $\begin{array}{l}\text { Sponso- } \\
\text { rengelder }\end{array}$ & $\begin{array}{l}\text { Vermögens- Sons- } \\
\text { erträge }\end{array}$ & tige \\
\hline bis 10000 & 49,1 & 14,8 & 19,2 & 5,2 & 3,4 & 2,6 & 49,1 \\
$10001-20000 €$ & 35,4 & 24,6 & 22,6 & 5,6 & 5,1 & 3,5 & 35,4 \\
$20001-100000 €$ & 29,1 & 25,0 & 21,6 & 11,6 & 5,8 & 4,3 & 29,1 \\
$100001-1$ Mio. $€$ & 20,5 & 23,7 & 13,8 & 30,7 & 3,7 & 4,3 & 20,5 \\
mehr als 1 Mio. $€$ & 6,8 & 40,8 & 11,5 & 32,8 & 1,9 & 3,4 & 6,8 \\
\hline Gesamt & 37,9 & 20,3 & 19,1 & 11,0 & 4,1 & 3,3 & 37,9 \\
\hline
\end{tabular}

Quelle: ZiviZ-Survey 2017: alle Organisationen, $\mathrm{n}=6750$ (gewichtet), davon fehlend: 1157 .

\subsection{Spenden ${ }^{4}$}

„Die einen spenden Zeit, die anderen Geld.“ geht eine landläufige Formulierung. Danach sind Spenden nicht einfach eine Finanzierungsquelle zivilgesellschaftlichen Handelns unter anderen. Spenden ist selbst eine Engagementform.

Spenden können in zahlreicher Hinsicht unterschieden werden. So können Sie zum einen von unterschiedlichen Akteuren stammen. Zu den wichtigsten zählen Privatpersonen und -haushalte, Unternehmen und gemeinnützige Stiftungen. Zum anderen lassen sich verschiedene Spendenformen unterscheiden. Denn spenden kann man nicht nur Geld. Gerade in der Phase der vermehrten Zuwanderung geflüchteter Menschen oder anderen krisenhaften Phasen im In- und Ausland kommt es vermehrt zu Kleider- und anderen Sachspenden. Auch lassen sich allgemeine Spenden und zweckgebundene Spenden unterscheiden. Letztere sind an bestimmte vom Spendenden formulierte Verwendungszwecke oder Auflagen geknüpft.

Abzugrenzen sind Spenden von der auf den ersten Blick ähnlichen Form des Sponsorings. Rechtlich gesehen handelt es sich beim Sponsoring um eine Tauschbeziehung, bei der das Gewähren eines geldwerten Vorteils im Gegenzug an die Erwartung gebunden ist, zum Beispiel die Kommunikations- und Marketingzie-

4 Dieses Unterkapitel wurde verfasst von Holger Krimmer, ZiviZ. 
le dieses Akteurs zu unterstützen. Damit entfällt das Moment der Freigebigkeit (ohne Gegenleistung), das für die Engagementform des Spendens konstitutiv ist.

Im Folgenden geht es nur um Spenden, die von außerhalb des gemeinnützigen Sektors an Akteure im gemeinnützigen Sektor fließen. Spenden interessiert damit als eine Finanzierungspraxis gemeinnützigen Handelns. Davon zu unterscheiden sind Zuwendungen zum Beispiel von gemeinnützigen Förderstiftungen, die wiederum finanzielle Stromgrößen innerhalb des gemeinnützigen Sektors sind.

\subsubsection{Spendenverhalten ${ }^{5}$}

Die Anzahl der SpenderInnen in Deutschland lag 2016 bei 22,1 Millionen Personen. Das sind 33 Prozent der deutschen Bevölkerung (Spendenquote), die im Jahr 2016 Geld an gemeinnützige Organisationen oder Kirchen gespendet haben. Diese Informationen stammen aus der „Bilanz des Helfens“, die die GfK im Auftrag des Deutschen Spendenrats e. V. erstellt und die ein Teilergebnis des GfK Panels Charity ${ }^{\star}$ Scope ist, das auf kontinuierlichen schriftlichen Erhebungen bei einer repräsentativen Stichprobe von 10 ooo Panelteilnehmern basiert. GfK Charity ${ }^{\star}$ Scope ermittelt fortlaufend Daten zum Spendenverhalten von privaten Verbrauchern in Deutschland. Unter anderem werden Spendenvolumen, Spendenhöhe und Spendenzwecke abgefragt. Als Spende zählen die von deutschen Privatpersonen freiwillig getätigten Geldspenden an gemeinnützige Organisationen, Hilfs- sowie Wohltätigkeitsorganisationen und Kirchen. Nicht enthalten sind Erbschaften und Unternehmensspenden, Spenden an politische Parteien und Organisationen und gerichtlich veranlasste Geldzuwendungen, Stiftungsneugründungen und Großspenden über 2500 Euro. Im Vorjahr waren es 22,7 Millionen SpenderInnen und damit rund 600 ooo Menschen mehr.

Der langfristige Vergleich zeigt, dass es im Jahr 2005 noch 34,6 Millionen Spender gab. Das entspricht einer Reichweite von über 50 Prozent, d.h. jeder Zweite (bezogen auf die Grundgesamtheit der privaten deutschen Personen ab zehn Jahren) hatte mindestens einmal pro Jahr eine Geldspende getätigt. Diese Reichweite ist in den Jahren 2005 bis 2009 dramatisch zurückgegangen und stagniert seit einigen Jahren auf diesem niedrigen Niveau. Nur in den Jahren, in denen es große Katastrophen gab, die medienwirksam unterstützt wurden, nahm die Anzahl der SpenderInnen wieder zu - wie zum Beispiel im Jahr 2010, in dem die Anzahl der SpenderInnen auf 24,6 Millionen kletterte (Hintergrund waren die Erdbebenkatastrophen in Haiti und in Pakistan). Allerdings wurde das hohe Niveau

5 Dieses Unterkapitel wurde verfasst von Bianca Corcoran-Schliemann, GfK und Daniela Geue, Deutscher Spendenrat. 
Tabelle 6.8 Spendenquote von 2005 bis 2016 (in Prozent)

\begin{tabular}{lllllllllllll}
\hline & 2005 & 2006 & 2007 & 2008 & 2009 & 2010 & 2011 & 2012 & 2013 & 2014 & 2015 & 2016 \\
\hline $\begin{array}{l}\text { Spenden- } \\
\text { quote }\end{array}$ & 50,9 & 42,6 & 40,6 & 40,1 & 34,3 & 36,1 & 32,4 & 33,2 & 34,3 & 33,0 & 33,6 & 32,7 \\
\hline
\end{tabular}

Quelle: Eigene Darstellung, GfK, Deutscher Spendenrat.

Tabelle 6.9 Spendenhäufigkeit von 2005 bis 2016 (in Prozent)

\begin{tabular}{|c|c|c|c|c|c|c|c|c|c|c|c|c|}
\hline & 2005 & 2006 & 2007 & 2008 & 2009 & 2010 & 2011 & 2012 & 2013 & 2014 & 2015 & 2016 \\
\hline $\begin{array}{l}\text { Spendenhäu- } \\
\text { figkeit durch- } \\
\text { schnittlich pro }\end{array}$ & $\begin{array}{l}4,3 \\
0\end{array}$ & 5,0 & 5,1 & 5,4 & 6,3 & 6,3 & 6,7 & 6,5 & 6,2 & 6,2 & 6,6 & 6,7 \\
\hline
\end{tabular}

Quelle: Eigene Darstellung, GfK, Deutscher Spendenrat.

von 2005 nie mehr erreicht. Im selben betrachteten Zeitraum nahm die Spendenhäufigkeit zu. Der Betrag einer durchschnittlichen Spende betrug im Jahr 2016 35 Euro, bei einer Spendenhäufigkeit von knapp sieben Spenden durchschnittlich pro Person und Jahr.

Die Spendenhäufigkeit nimmt im langfristigen Vergleich zu, obwohl die Anzahl der SpenderInnen über die Jahre deutlich zurückgeht. Das bedeutet, dass es Personen oder Gruppen von Personen gibt, die regelmäßig hohe Beträge spendeten. Für diese Menschen gehört das Spenden zur Selbstverständlichkeit. Was sind die Hintergründe?

Die zahlenmäßig größte Gruppe der SpenderInnen macht die Altersgruppe $70+$ aus.

Im Jahr 2016 lag der Anteil der SpenderInnen in dieser Altersgruppe bei 57 Prozent, das heißt, über die Hälfte der Personen, die älter als 70 Jahre sind, spendeten mindestens einmal pro Jahr. Dieser Anteil nahm in den letzten fünf Jahren zu. So lag er im Jahr 2015 bei 55 Prozent und im Jahr 2012 bei 54 Prozent. Auch spendete diese Altersgruppe 2016 mit durchschnittlich rund 290 Euro im Jahr am meisten. Die jüngeren Altersgruppen waren nicht so spendenfreudig. In der Altersgruppe 60 bis 69 Jahre spendeten nur rund 39 Prozent, gemessen an der privaten deutschen Bevölkerung ab zehn Jahren. Das durchschnittliche Spendenvolumen lag in dieser Altersgruppe bei 237 Euro pro Jahr. Bei den 50- bis 59-Jährigen lag die Spenderreichweite in 2016 mit einem durchschnittlichen Spendenvolumen von 224 Euro nur noch bei 31 Prozent. Die generelle Altersentwicklung in der 
Tabelle 6.10 SpenderInnenanteil innerhalb der Altersgruppe, 2016 (in Prozent)

\begin{tabular}{lllll}
\hline 2016 & $\begin{array}{l}\text { Bevölkerung } \\
\text { in Mio. EW }\end{array}$ & Anteil in \% & $\begin{array}{l}\text { Spenderlnnen } \\
\text { in Mio. }\end{array}$ & $\begin{array}{l}\text { Anteil Spender- } \\
\text { Innen in \% in } \\
\text { Altersgruppe }\end{array}$ \\
\hline TOTAL & $67,7^{*}$ & 100 & 22,1 & 33 \\
bis 29 Jahre & 15,1 & 22 & 2,3 & 15 \\
30 bis 49 Jahre & 18,7 & 28 & 5,3 & 28 \\
50 bis 59 Jahre & 12,0 & 18 & 3,7 & 31 \\
60 bis 69 Jahre & 9,0 & 13 & 3,5 & 39 \\
70 Jahre und älter & 12,9 & 19 & 7,4 & 57 \\
\hline
\end{tabular}

Quelle: Eigene Darstellung, GfK, Deutscher Spendenrat.

* Bevölkerung ab dem 10. Lebensjahr

Tabelle 6.11 Spendenvolumen in der jeweiligen Altersgruppe, 2015-2016 (in Prozent)

\begin{tabular}{lcc}
\hline & 2015 & 2016 \\
\hline bis 29 Jahre & 4,0 & 5,1 \\
30 bis 49 Jahre & 27,2 & 23,1 \\
50 bis 59 Jahre & 14,2 & 15,6 \\
60 bis 69 Jahre & 15,5 & 15,7 \\
70 Jahre und älter & 39,1 & 40,4 \\
\hline
\end{tabular}

Quelle: Eigene Darstellung, GfK, Deutscher Spendenrat.

Bevölkerung zeigt in den letzten fünf Jahren eine deutliche Zunahme der Bevölkerung in der Altersgruppe 50 bis 59 (plus 13 Prozent), ein leichtes Minus von 2 Prozent in der Gruppe der 6o-bis 69-Jährigen. Die Anzahl der SpenderInnen geht aber im selben Zeitraum bei den 50- bis 59-Jährigen um 3 Prozent, bei den 6o- bis 69-Jährigen sogar um 15 Prozent zurück. Es sind die Babyboomer - die Geburtsjahrgänge 1952 bis 1966 -, aufgewachsen in einer Zeit, in der viele vom ewigen Wohlstand träumten. Es ging immer aufwärts. Es gab keine Sorgen um Arbeitslosigkeit, Rente etc. Sie zeichnen sich durch Sorgenfreiheit und klare Rollentrennung aus. Gerade diese beiden Altersgruppen sind zum Teil wirtschaftlich gut gestellt. Es handelt sich bei ihnen vorwiegend um Ein- bis Zwei-Personen-Haushalte, deren Nettohaushaltseinkommen in den Einkommensklassen 1750 Euro bis 
2249 Euro, 2250 Euro bis 2749 Euro und insbesondere 3250 Euro bei 3749 Euro überproportional hoch zu allen SpenderInnen ist. Diese deutlich bessere wirtschaftliche Situation führt bei Babyboomern aber nicht zu einem Anstieg des Spendenvolumens. Spenden steht bei diesen Generationen nicht mehr so im Fokus wie noch bei der Wiederaufbaugeneration. Um die Gründe für diese Spendenträgheit $\mathrm{zu}$ analysieren, stehen aus dem GfK Charity ${ }^{\star}$ Scope weiterführende Informationen zur Verfügung. Ein Kriterium ist, ob diese Menschen zum Gottesdienst gehen oder nicht. SpenderInnen für kirchliche Belange sind zum überwiegenden Teil Menschen, die zumindest gelegentlich Gottesdienste besuchen. Dabei ist die Verbindung zwischen Gottesdienstbesuch und Spende für die Kirche in der katholischen Kirche noch stärker ausgeprägt als in der evangelischen. Im Umkehrschluss heißt das aber auch, dass eine sinkende Zahl von Kirchenbesuchern quasi automatisch auch einen Rückgang des Spendenvolumens nach sich zieht.

Die Spenderreichweite der 30- bis 49-Jährigen ist im Vergleich zur Verteilung in der Gesamtbevölkerung deutlich unterproportional. Hinzu kommt: Wenn sie spenden, dann deutlich weniger als die älteren Altersgruppen. Der eine spendet Geld, der andere Zeit. So hat die GfK aus dem Charity ${ }^{\star}$ Scope in der Vergangenheit analysieren können, dass GeldspenderInnen meist die über 6o-Jährigen sind und die ZeitspenderInnen sich eher in den jüngeren Zielgruppen wiederfinden. Überproportional viele ältere Menschen (im Vergleich zur Gruppe aller SpenderInnen) halten das Spenden für selbstverständlich (Index 107), sie wünschen sich genaue Informationen über die Spendenprojekte (Index 103) und fühlen sich durch die Vielzahl an ähnlichen Organisationen etwas überfordert (Index 103).

Tabelle 6.12 Gottesdienst-Spendenvolumen 2015 bis 2016 (in Prozent)

\begin{tabular}{lll}
\hline & \multicolumn{2}{l}{ Spendenvolumen } \\
\cline { 2 - 3 } & 2015 & 2016 \\
\hline Gottesdienst Nennungen & 100 & 100 \\
Gottesdienst häufig & 21,9 & 26,9 \\
Gottesdienst selten & 22,1 & 29,6 \\
Gottesdienst nie & 25,1 & 21,2 \\
Gottesdienst & 21,6 & 15,6 \\
gelegentlich & & \\
Rest & 9,4 & 6,8 \\
\hline
\end{tabular}

Quelle: Eigene Darstellung, GfK, Deutscher Spendenrat. 
Aber es gibt auch Überschneidungen. Geldspende und Zeitspende schließen sich nicht gegenseitig aus. Jüngere Menschen engagieren sich eher ehrenamtlich und sind bereit, ihre Zeit für gemeinnützige Zwecke einzubringen. Dass Jugendliche dabei ihren Fokus anders legen als Erwachsene, kann nicht zuletzt durch die Erwerbsfähigkeit erklärt werden. Jugendliche zum Beispiel sind als ehrenamtliche MitarbeiterInnen in der Kirche, in der Kinder- und Jugendarbeit, im Bereich Bildung, Rettungswesen und natürlich auch im Sport besonders stark. Zahlreiche weiterführende Informationen helfen dabei, diese Gruppen im Detail zu verstehen.

\subsubsection{Spendenzwecke ${ }^{6}$}

Die Entwicklung des Spendenvolumens ist seit 2005, trotz Rückgangs der Anzahl der SpenderInnen, aufgrund der wachsenden Häufigkeit und Intensität positiv.

Der größte Anteil des Spendenvolumens fließt in die humanitäre Hilfe. Dieser Anteil lag im Jahr 2016 bei 77 Prozent und damit etwas unter dem des Vorjahres. Man kann die humanitäre Hilfe weiter unterteilen. 2o Prozent der Spendeneinnahmen für die humanitäre Hilfe werden für Kirche/Religion, 21 Prozent für die Kinder- und Jugendhilfe, 12 Prozent für die Not- und Katastrophenhilfe, 9 Prozent für die Unterstützung bei Krankheit und Behinderung und 14 Prozent für sonstige humanitäre Hilfe, zu der zum Beispiel die langfristige Entwicklungshilfe, Bildung und sonstige soziale Zwecke zählen, gespendet. Gerade in Jahren einer medienwirksamen Katastrophe zeigen die Zahlen aus dem GfK Charity ${ }^{\star}$ Scope deutliche Anstiege im Bereich der humanitären Hilfe (zum Beispiel bei der Katastrophenhilfe). Hierbei kann die GfK monatsgenau belegen, wie viel gespendet wurde. So wird deutlich, dass es immer wieder der Monat Dezember ist, der einen großen Anteil am Jahresspendenaufkommen hat.

23 Prozent der Spenden flossen 2016 in die nicht humanitäre Hilfe. Darunter fallen Kultur- und Denkmalpflege, Umwelt- und Naturschutz, Tierschutz und Spenden für Sport. Rückgänge im Spendenaufkommen verzeichnete im Jahr 2016 vor allem der Bereich humanitäre Hilfe. Daneben musste auch der Tierschutz ein leichtes Minus verbuchen. Kultur-/Denkmalpflege und Umwelt-/Naturschutz konnten dagegen ein leichtes Plus verzeichnen. Einen deutlich geringeren Anteil am Spendenvolumen hatte die Not- und Katastrophenhilfe. Der Anteil des Spendenaufkommens für die Katastrophenhilfe lag 2016 deutlich unter dem Niveau von 2015. Hier wurden 32 Prozent weniger als im Vorjahr gespendet. Sowohl die

6 Dieses Unterkapitel wurde verfasst von Bianca Corcoran-Schliemann, GfK und Daniela Geue, Deutscher Spendenrat. 
Tabelle 6.13 Spendenvolumen 2005 bis 2016 (in Prozent)

\begin{tabular}{|c|c|c|c|c|c|c|c|c|c|c|c|c|}
\hline & 2005 & 2006 & 2007 & 2008 & 2009 & 2010 & 2011 & 2012 & 2013 & 2014 & 2015 & 2016 \\
\hline $\begin{array}{l}\text { Spenden- } \\
\text { volumen } \\
\text { Index } 2005= \\
100 \%\end{array}$ & 100 & 84 & 85 & 88 & 92 & 99 & 93 & 91 & 103 & 108 & 121 & 115 \\
\hline
\end{tabular}

Quelle: Eigene Darstellung: GfK, Deutscher Spendenrat.

Tabelle 6.14 Spendenvolumen nach Verteilung der Spendenzwecke 2014 bis 2016 (in Prozent)

\begin{tabular}{llll}
\hline & \multicolumn{3}{l}{ Spendenvolumen } \\
\cline { 2 - 4 } & 2014 & 2015 & 2016 \\
\hline Humanitäre Hilfe & 79,0 & 79,3 & 76,8 \\
Hilfe für Kinder & 20,9 & 20,7 & 21,0 \\
Kirchl./religiöse Zwecke & 19,9 & 18,4 & 20,4 \\
Not-/Katastrophenhilfe & 11,9 & 16,5 & 11,8 \\
Hilfe für Kranke/Behinderte & 11,5 & 7,9 & 9,4 \\
$\quad$ Entwicklungshilfe & 3,8 & 2,8 & 3,6 \\
$\quad$ andere soziale Zwecke & 11,0 & 13,0 & 10,6 \\
Tierschutz & 5,7 & 5,3 & 5,5 \\
Sport & 2,4 & 2,5 & 2,7 \\
Kultur-/Denkmalpflege & 2,9 & 2,4 & 2,6 \\
Umwelt-/Naturschutz & 2,7 & 2,1 & 2,5 \\
Sonstige/Keine Angaben & 7,4 & 8,4 & 10,0 \\
\hline
\end{tabular}

Quelle: Eigene Darstellung: GfK, Deutscher Spendenrat.

Anzahl der SpenderInnen, die Anzahl der Spendenakte als auch die durchschnittliche Spendenhöhe pro Spendenakt gingen zurück.

Das liegt unter anderem daran, dass 2015 das Thema Flüchtlingswanderung stark in der öffentlichen Diskussion stand und die Linderung der Erdbebenkatastrophe in Nepal stark unterstützt wurde. Insgesamt 488 Millionen Euro - das sind 9 Prozent des Spendenvolumens - flossen im Jahr 2016 in die Hilfe für Flüchtlinge. Fast vier Millionen Personen, das sind 17 Prozent aller SpenderInnen, also 
Tabelle 6.15 Durchschnittliches Spendenvolumen, Spendenakt und SpenderInnen 2005 bis 2016

\begin{tabular}{|c|c|c|c|c|c|c|c|c|c|c|c|c|}
\hline & 2005 & 2006 & 2007 & 2008 & 2009 & 2010 & 2011 & 2012 & 2013 & 2014 & 2015 & 2016 \\
\hline $\begin{array}{l}\text { Spenderlnnen } \\
\text { (in Tsd.) }\end{array}$ & 34,6 & 29,0 & 27,6 & 27,3 & 23,3 & 24,6 & 22,0 & 22,5 & 23,3 & 22,4 & 22,7 & 22,1 \\
\hline $\begin{array}{l}\text { durchschnitt- } \\
\text { liches Spen- } \\
\text { denvolumen } \\
\text { pro Spende (in } \\
\text { Euro) }\end{array}$ & 30,9 & 26,7 & 27,7 & 27,5 & 28,6 & 29,3 & 29,0 & 28,6 & 32,6 & 35,5 & 37,2 & 35,5 \\
\hline $\begin{array}{l}\text { Spendenakte } \\
\text { (in Tsd.) }\end{array}$ & 148,4 & 144,4 & 140,9 & 147,2 & 147,2 & 154,5 & 146,6 & 145,2 & 144,3 & 139,6 & 149,0 & 148,4 \\
\hline
\end{tabular}

Quelle: Eigene Darstellung: GfK, Deutscher Spendenrat.

knapp 6 Prozent aller Deutschen über zehn Jahre, spendeten für Flüchtlinge im Inund Ausland. Dabei entfielen 57 Prozent des Gesamtvolumens auf Flüchtlingsprojekte in Deutschland und 43 Prozent auf internationale Flüchtlingsprojekte. Mit 46 Euro lag das Spendenvolumen deutlich höher als die Durchschnittspende im Markt. Nachdem weiter oben dargestellt wurde, dass die Anzahl der GeldspenderInnen (nicht das freiwillige Engagement) in den jüngeren Zielgruppen eher gering ist, zeigen die diesbezüglichen Analysen beim Flüchtlingsthema, dass gerade diese jungen Zielgruppen einen überdurchschnittlichen Anteil am entsprechenden Spendenbudget aufweisen.

\subsubsection{Finanzstruktur von Spendenorganisationen ${ }^{7}$}

Daten zur Finanzstruktur und zu anderen Merkmalen von Spendenorganisationen lassen sich der jährlichen Statistik des Deutschen Zentralinstituts für soziale Fragen (DZI) entnehmen. Die Statistik erscheint im DZI Spenden-Almanach, der jährlich im November/Dezember veröffentlicht wird. Die Daten im statistischen Anhang des Spenden-Almanachs beziehen sich in erster Linie auf die Organisationen, die mit dem DZI Spenden-Siegel ausgezeichnet wurden. Die Datenerhebung nimmt das DZI im Rahmen der jährlichen Spenden-Siegel-Prüfungen vor. Grundlage sind unter anderem die Jahresabschlüsse und die Wirtschaftsprü-

7 Dieses Unterkapitel wurde verfasst von Karsten Schulz-Sandhof, DZI. 
fungsberichte der Organisationen sowie die Gliederungsmerkmale und Definitionen, wie sie in den Leitlinien des Spenden-Siegels und im DZI-Konzept zur Berechnung der Werbe- und Verwaltungsausgaben Spenden sammelnder Organisationen niedergelegt sind.

Die Statistik für die Gesamtheit der Spenden-Siegel-Organisationen liegt verfahrensbedingt etwa zweieinhalb Jahre nach dem jeweiligen Berichtsjahr vor. Aktuell ist derzeit die Statistik für das Jahr 2014, veröffentlicht im Spenden-Almanach 2017. Der Statistik liegen die Daten der 227 Organisationen zugrunde, die im Dezember 2017 das Spenden-Siegel führten.

Das Spektrum der Spenden-Siegel-Organisationen ist breit gefächert. Es umfasst kleine bis sehr große Organisationen verschiedener Rechtsformen (vor allem Vereine), die in unterschiedlichsten Bereichen sowohl im Inland als auch im Ausland tätig sind. Die Spenden-Siegel-Organisationen erzielten im Jahr 2014 Einnahmen von insgesamt 4,4 Milliarden Euro, darunter Geldspenden in Höhe von 1,4 Milliarden Euro. Dies entspricht nach den Berechnungen des DZI etwa 18 Prozent des gesamten Geldspendenaufkommens in Deutschland. Die Gesamtheit der Spenden-Siegel-Organisationen kann aufgrund ihrer heterogenen Zusammensetzung und ihres Finanzvolumens als Spiegel des deutschen Spendensektors gesehen werden, auch wenn sich das Spenden-Siegel nicht auf sehr kleine Organisationen mit jährlichen Gesamteinnahmen von weniger als 25 ooo Euro bezieht.

Die Geldspenden umfassen nach der Definition des DZI die Geldspenden von privaten Personen und Unternehmen einschließlich Einnahmen aus Kollekten, Patenschaftsbeiträgen und Fördermitgliedsbeiträgen. Nicht zu den Geldspenden zählen unter anderem Mitgliedsbeiträge, Nachlässe, Geldauflagen und Zuwendungen anderer gemeinnütziger Organisationen. Die nachstehende Tabelle gibt einen Überblick über die aggregierten Einnahmen, Ausgaben und das Vermögen der Spenden-Siegel-Organisationen im Jahr 2014. Das prozentuale Gewicht der einzelnen Posten ändert sich von Jahr zu Jahr nur in geringem Maße.

Die wesentlichen Finanzierungsquellen der Spenden-Siegel-Organisationen sind Geldspenden (31,o Prozent der Gesamteinnahmen), Leistungsentgelte (27,7 Prozent) und Mittel der öffentlichen Hand (19,7 Prozent), gefolgt von Zuwendungen anderer gemeinnütziger Organisationen (7,4 Prozent) und Nachlässen (4,6 Prozent). Die weiteren Einnahmen, darunter Sachspenden, Mitgliedsbeiträge und Geldauflagen, spielen in der Gesamtbetrachtung quantitativ nur eine untergeordnete Rolle. Individuell ist das bei den Organisationen natürlich sehr unterschiedlich.

Die Ausgaben der Spenden-Siegel-Organisationen beziehen sich zu 86,4 Prozent auf die Verwirklichung des unmittelbar satzungsgemäßen Zwecks. Die übrigen Ausgaben beinhalten die Werbe- und Verwaltungsausgaben sowie die Ausgaben für Vermögensverwaltung und den steuerpflichtigen Geschäftsbetrieb. 
Das aggregierte Vermögen der Spenden-Siegel-Organisationen besteht zum größten Teil aus Finanzvermögen (58,2 Prozent). Ein Teil des Vermögens gehört zum Vermögensstock der Organisationen, der nach Maßgabe des Gemeinnützigkeitsrechts dauerhaft zur Erzielung von Erträgen gehalten werden darf bzw. - vor allem bei Stiftungen - gehalten werden muss. Die Finanzierung des dauerhaften Vermögens geht insbesondere auf entsprechend zweckgebundene Spenden und Nachlässe bzw. auf Stiftungen und Zustiftungen zurück.

Neben Finanzmitteln erhalten Spendenorganisationen in umfangreicher und vielfältiger Weise kostenlose Leistungen. $\mathrm{Zu}$ nennen ist hier an erster Stelle das ehrenamtliche Engagement sehr vieler Menschen. Hinzu kommen unentgeltliche Sach- und Dienstleistungen, zum Beispiel die kostenlose Zurverfügungstellung von Material, Räumen und Serviceleistungen durch Privatpersonen, Kirchen und Unternehmen. All diese Leistungen, die in einem erweiterten Sinn meist auch als Spenden zu betrachten sind, schlagen sich in der Regel nicht in den Jahresabschlüssen der Organisationen nieder.

Tabelle 6.16 Finanzdaten 2014 der Spenden-Siegel-Organisationen

\begin{tabular}{|c|c|c|c|c|c|}
\hline Einnahmen & Mio. Euro & In Prozent & Ausgaben & Mio. Euro & In Prozent \\
\hline Geldspenden & 1366 & 31,0 & Projektausgaben & 3766 & 86,4 \\
\hline Leistungsentgelte & 1221 & 27,7 & Werbeausgaben & 248 & 5,7 \\
\hline $\begin{array}{l}\text { Öffentliche Zuwen- } \\
\text { dungen }\end{array}$ & 866 & 19,7 & $\begin{array}{l}\text { Verwaltungsaus- } \\
\text { gaben }\end{array}$ & 248 & 5,7 \\
\hline $\begin{array}{l}\text { Zuwendungen von } \\
\text { Organisationen }\end{array}$ & 326 & 7,4 & $\begin{array}{l}\text { Vermögensverwal- } \\
\text { tung }\end{array}$ & 56 & 1,3 \\
\hline Nachlässe & 201 & 4,6 & $\begin{array}{l}\text { Steuerpflichtiger Ge- } \\
\text { schäftsbetrieb }\end{array}$ & 39 & 0,9 \\
\hline $\begin{array}{l}\text { Zins- und Vermö- } \\
\text { genseinnahmen }\end{array}$ & 105 & 2,4 & Gesamtausgaben & 4357 & 100,0 \\
\hline Mitgliedsbeiträge & 88 & 2,0 & Vermögen & Mio. Euro & In Prozent \\
\hline Sachspenden & 47 & 1,1 & Finanzvermögen & 2776 & 58,2 \\
\hline Geldauflagen & 12 & 0,3 & Sachvermögen & 1474 & 30,9 \\
\hline Sonstige Einnahmen & 171 & 3,9 & Sonstiges Vermögen & 516 & 10,8 \\
\hline Gesamteinnahmen & 4403 & 100,0 & Gesamtvermögen & 4766 & 100,0 \\
\hline
\end{tabular}

Quelle: Eigene Darstellung, DZI. 


\subsubsection{Spendenvolumen und Spendenquote ${ }^{8,9}$}

Daten zum Geldspendenvolumen in Deutschland werden von verschiedenen Institutionen erhoben. Tabelle 6.17 gibt einen grundlegenden Überblick über die Erhebungen, die beteiligten Institutionen sowie über Art und Intervall der Durchführung.

Die Erhebungen kommen in Bezug auf das Geldspendenvolumen zu unterschiedlichen Ergebnissen. Für einen Vergleich eignet sich das Jahr 2014, da für dieses Jahr - mit Ausnahme der Lohn- und Einkommensteuerstatistik - die Daten von allen Erhebungen vorliegen.

Die Daten aus dem Sozio-oekonomischen Panel (SOEP) ergeben für 2014 ein Geldspendenvolumen von 8,o Milliarden Euro. Demgegenüber kommt der Deutsche Spendenmonitor für dasselbe Jahr auf weniger als die Hälfte (3,7 Milliarden Euro). Die Bilanz des Helfens liegt bei 5,o Milliarden Euro. Der Deutsche Freiwilligensurvey ermittelt 5,9 Milliarden Euro. Das Deutsche Zentralinstitut für soziale Fragen (DZI), das auf der Grundlage seines Spenden-Index die Hochrechnung aus dem SOEP jährlich fortschreibt, kommt für 2014 - ausgehend noch von der SOEPErhebung für 2009 - auf ein Gesamtvolumen von 6,7 Milliarden Euro.

Unterschiedliche Ergebnisse liegen auch für die Spendenquote vor, also für den Anteil der SpenderInnen an der Gesamtbevölkerung. Hier liegt die Spanne zwischen 33 Prozent (Bilanz des Helfens) und 54 Prozent (Freiwilligensurvey). Spendenmonitor und SOEP liegen mit 45 Prozent bzw. 47 Prozent im Jahr 2014 nah beieinander. Lediglich angemerkt sei an dieser Stelle, dass die verschiedenen Umfragen in ihren differenzierteren Analysen zum Spendenverhalten (etwa nach Alter, Geschlecht, Bildung und Einkommen) im Grundsatz zu sehr übereinstimmenden Ergebnissen kommen. Demnach spenden Ältere mehr als Jüngere, Frauen mehr als Männer, höher Gebildete mehr als gering Gebildete und Einkommensstarke mehr als Einkommensschwache.

Die Abbildungen 6.2 und 6.3 zeigen die Entwicklungen des Spendenvolumens und der Spendenquote ab dem Jahr 2009. Dieses Jahr bietet sich - auch wenn es durch die Finanzkrise möglicherweise negativ beeinflusst ist - als Beginn der Zeitreihe an, da für 2009 erstmals auch Spendendaten aus dem SOEP vorliegen. Die Spendenfrage wurde im SOEP nach 2009 wieder für das Jahr 2014 gestellt. Der Freiwilligensurvey wird seit 1999 alle fünf Jahre erhoben. Sowohl das SOEP als

8 Der Beitrag wurde ursprünglich in ähnlicher Fassung unter dem Titel „Wie viel wird in Deutschland gespendet - 4 oder 8 Milliarden Euro?“" in der elektronischen Schriftenreihe Observatorium der Maecenata Stiftung veröffentlicht (Ausgabe 17/2017). Zudem ist der Beitrag in ähnlicher Fassung im DZI Spenden-Almanach 2017 erschienen.

9 Dieses Unterkapitel wurde verfasst von Karsten Schulz-Sandhof, DZI. 


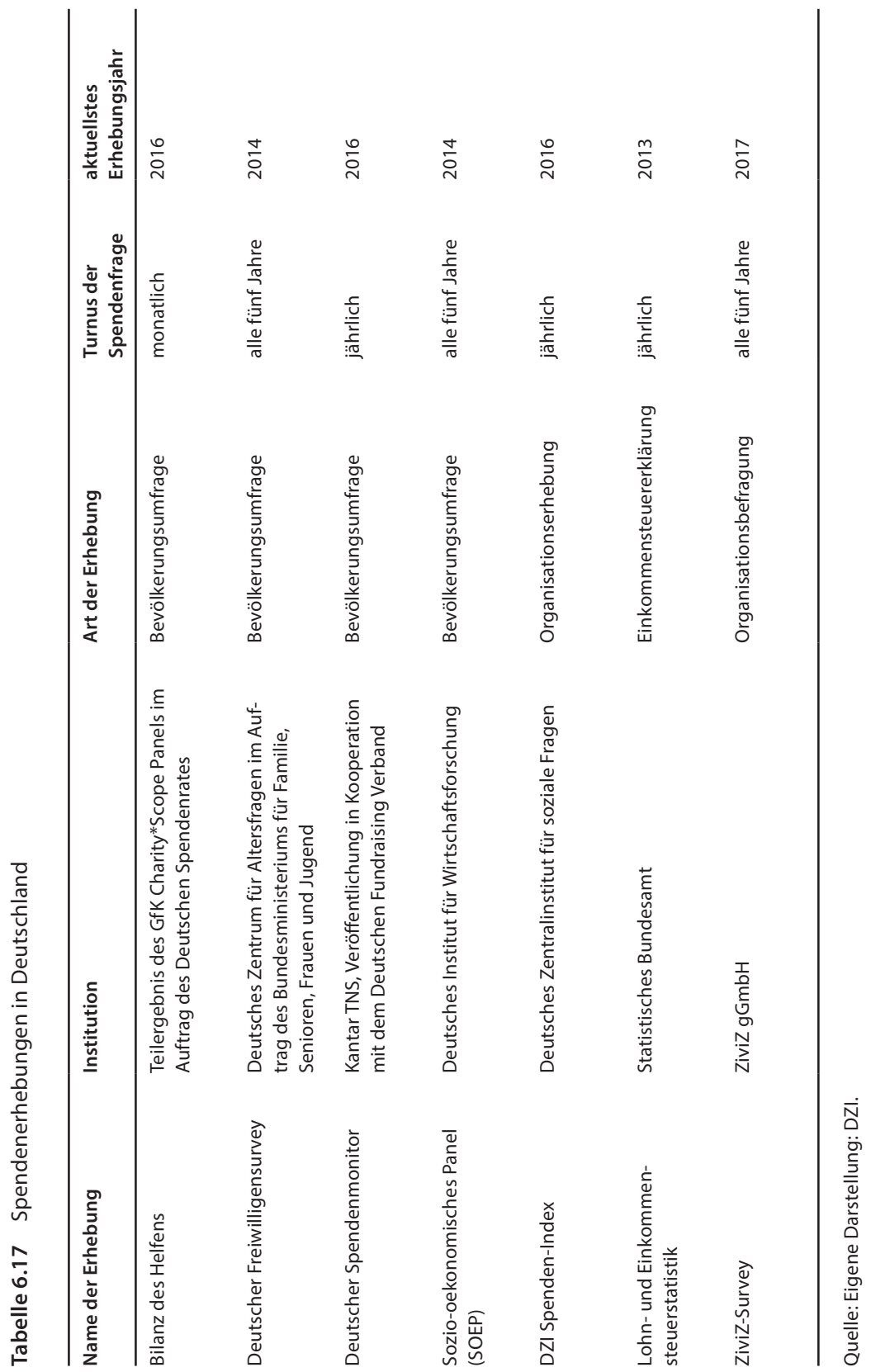


Abbildung 6.2 Geldspendenvolumen in Deutschland (in Milliarden Euro)

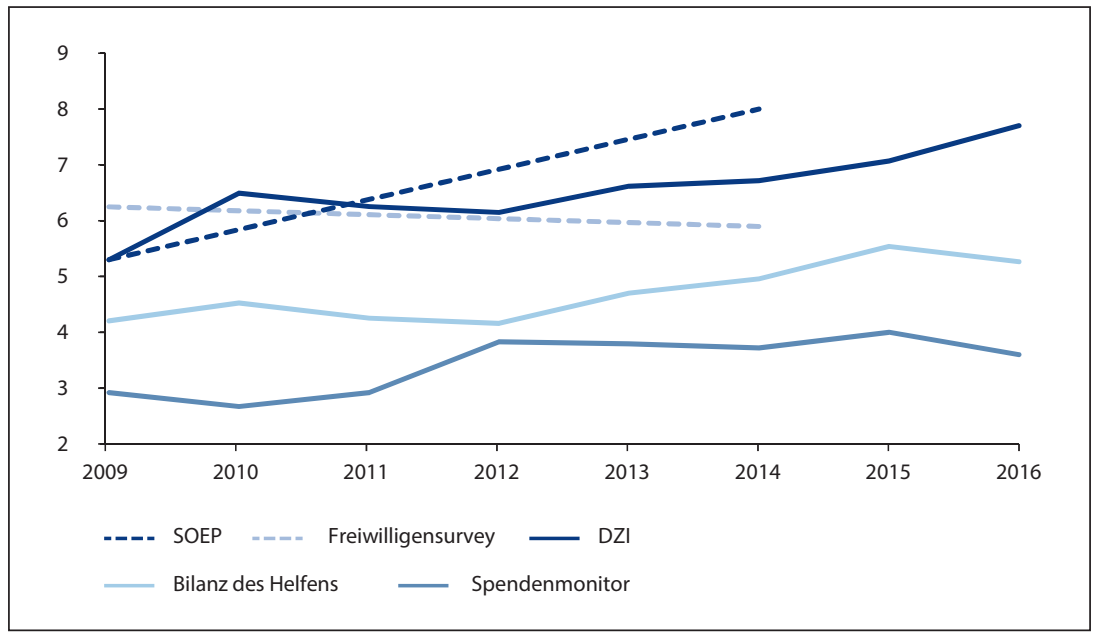

Quelle: Eigene Darstellung: DZI auf Basis der von den Erhebungen jeweils veröffentlichten Daten.

Abbildung 6.3 Spendenquote in Deutschland (in Prozent)

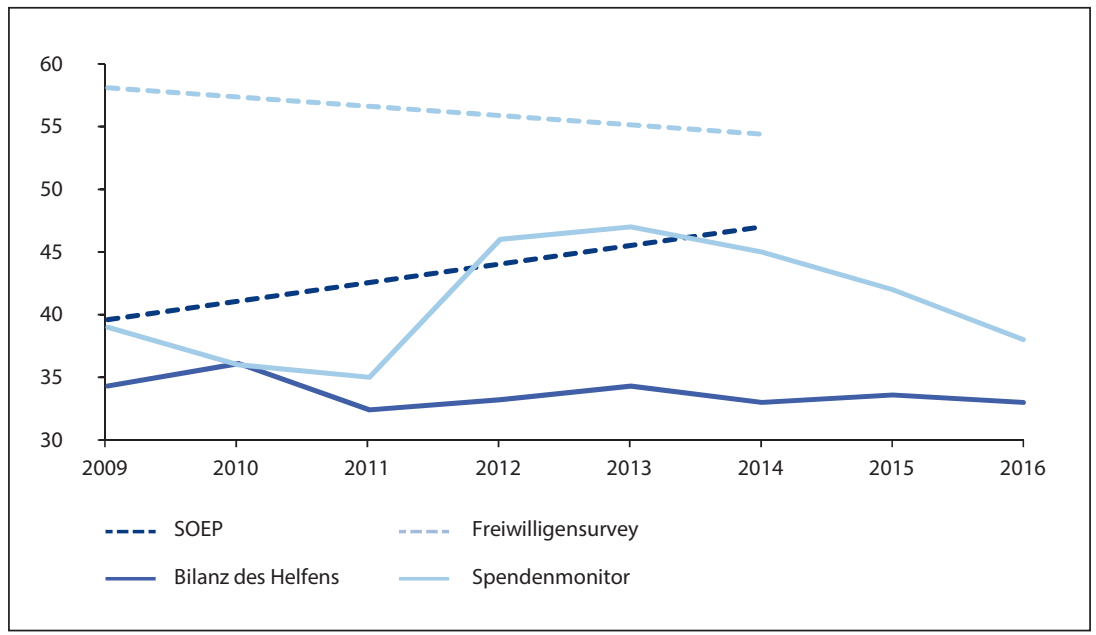

Quelle: Eigene Darstellung: DZI auf Basis der von den Erhebungen jeweils veröffentlichten Daten. 
auch der Freiwilligensurvey liefern somit Daten für 2009 und 2014. Die gestrichelt gehaltenen Linien dieser beiden Erhebungen verbinden in den beiden Schaubildern also nur zwei Punkte. Die anderen Erhebungen veröffentlichen seit vielen Jahren jährlich Ergebnisse.

Ausgestaltung der Bevölkerungsumfragen: Aus welchem Grund kommen die Bevölkerungsumfragen trotz repräsentativer Stichproben zu unterschiedlichen Ergebnissen? Die Antwort liegt grundsätzlich in den verschiedenen Erhebungs- und Berechnungsmethoden. Mit einem näheren Blick auf die Verfahren lässt sich den Faktoren ein wenig auf die Spur kommen. Die Tabellen 6.18 und 6.19 geben einen Überblick über die Ausgestaltungen der Umfragen und die Formulierung der jeweiligen Spendenfrage bzw. der zugrunde liegenden Spendendefinition.

Die Grundgesamtheit ist mit Ausnahme des Mindestalters in allen Umfragen mehr oder weniger gleich. Es geht im Kern um eine Erfassung der Spendentätigkeit der Bevölkerung in Deutschland. Unterschiede bestehen vor allem darin, ob nur deutschsprachige oder auch fremdsprachige Personen befragt werden. Das Mindestalter der Befragten wird bei dem der Bilanz des Helfens zugrunde liegenden GfK Charity ${ }^{\star}$ Scope mit zehn Jahren am niedrigsten angesetzt, beim SOEP ist es mit 18 Jahren am höchsten.

Bei der Bilanz des Helfens und beim SOEP beziehen sich die Umfragen jeweils auf einen feststehenden Personenkreis (Panel). Demgegenüber werden die befragten Personen beim Spendenmonitor und beim Freiwilligensurvey bei jeder Befragung in einem statistisch repräsentativen Zufallsverfahren neu ausgewählt (Welle). Unterschiede bestehen bei allen Umfragen im Hinblick auf den Stichprobenumfang. Die Spanne liegt zwischen 4 ooo Personen (Spendenmonitor) und 29000 Personen (Freiwilligensurvey 2014).

Auch die Art der Auskunftserteilung durch die Befragten unterscheidet sich. Der Freiwilligensurvey stellt die Fragen auf telefonischem Wege, beim Spendenmonitor werden persönliche Interviews geführt, bei dem der Bilanz des Helfens zugrunde liegenden Verfahren führen die TeilnehmerInnen ein schriftliches, monatliches Tagebuch. Der SOEP-Fragebogen wird schriftlich oder persönlich beantwortet. Die Form und das Intervall der Befragung könnten einen unterschiedlichen Einfluss auf die Genauigkeit der Angaben haben. Eine Rolle dürfte dabei auch spielen, dass Spenden als sozial erwünscht angesehen werden.

Abweichungen bestehen zudem beim zeitlichen Bezug der Spendenfragen. Die Bilanz des Helfens bezieht sich auf kontinuierliche, monatliche Spendenangaben. Das SOEP fragt nach den Spenden im letzten Kalenderjahr. Demgegenüber geht es beim Freiwilligensurvey und beim Spendenmonitor um die Spenden in den letzten zwölf Monaten. Die Antworten der Befragten beziehen sich bei diesen beiden Umfragen also auf Zeiträume, die innerhalb von zwei Kalenderjah- 
Tabelle 6.18 Merkmale der Bevölkerungsumfragen in Deutschland zum Spendenaufkommen

\begin{tabular}{|c|c|c|c|c|}
\hline & $\begin{array}{l}\text { Bilanz des } \\
\text { Helfens }\end{array}$ & $\begin{array}{l}\text { Freiwilligen- } \\
\text { survey }\end{array}$ & SOEP & $\begin{array}{l}\text { Spenden- } \\
\text { monitor }\end{array}$ \\
\hline Grundgesamtheit & $\begin{array}{l}\text { deutsche Privat- } \\
\text { personen in } \\
\text { Deutschland }\end{array}$ & $\begin{array}{l}\text { Wohnbevölke- } \\
\text { rung Deutsch- } \\
\text { lands }\end{array}$ & $\begin{array}{l}\text { Privatpersonen } \\
\text { in Deutschland }\end{array}$ & $\begin{array}{l}\text { deutschsprachi- } \\
\text { ge Bevölkerung } \\
\text { in Deutschland }\end{array}$ \\
\hline $\begin{array}{l}\text { Stichprobe } \\
\text { (gerundet) }\end{array}$ & 10000 Personen & 29000 Personen & 17000 Personen & 4000 Personen \\
\hline $\begin{array}{l}\text { Panel (fester } \\
\text { Personenkreis) }\end{array}$ & ja & nein & ja & nein \\
\hline $\begin{array}{l}\text { Mindestalter der } \\
\text { befragten Personen }\end{array}$ & 10 Jahre & 14 Jahre & 18 Jahre & 14 Jahre \\
\hline Erhebungsintervall & monatlich & alle fünf Jahre & alle fünf Jahre & jährlich \\
\hline Form der Angaben & $\begin{array}{l}\text { schriftlich (Tage- } \\
\text { buch) }\end{array}$ & telefonisch & $\begin{array}{l}\text { schriftlich oder } \\
\text { persönlich }\end{array}$ & persönlich \\
\hline Frage nach & $\begin{array}{l}\text { konkretem } \\
\text { Spendenbetrag }\end{array}$ & $\begin{array}{l}\text { bestimmten } \\
\text { Spenden- } \\
\text { kategorien }\end{array}$ & $\begin{array}{l}\text { konkretem } \\
\text { Spendenbetrag }\end{array}$ & $\begin{array}{l}\text { konkretem } \\
\text { Spendenbetrag }\end{array}$ \\
\hline $\begin{array}{l}\text { Spendenkappung } \\
\text { bei Hochrechnung }\end{array}$ & ab 2500 Euro & ab 2000 Euro & keine Kappung & $\begin{array}{l}\text { ohne außer- } \\
\text { ordentliche } \\
\text { Großspenden }\end{array}$ \\
\hline $\begin{array}{l}\text { Zeitlicher Bezug der } \\
\text { Spendenfrage }\end{array}$ & Kalenderjahr & letzte 12 Monate & Kalenderjahr & letzte 12 Monate \\
\hline $\begin{array}{l}\text { Spendenvolumen } \\
2014\end{array}$ & 5,0 Mrd. Euro & 5,9 Mrd. Euro & 8,0 Mrd. Euro & 3,7 Mrd. Euro \\
\hline Spendenquote 2014 & $33 \%$ & $54 \%$ & $47 \%$ & $45 \%$ \\
\hline
\end{tabular}

Quelle: Eigene Darstellung: DZI auf Basis der von den Erhebungen jeweils veröffentlichten Angaben.

ren liegen. Beim Freiwilligensurvey ist die Mischung besonders ausgeprägt, weil die Befragung von etwa Mai bis Oktober 2014 stattfand. Dies kann je nach zeitlicher Lage zu gewissen Verzerrungen führen, wenn Notlagen oder Katastrophen eine außerordentliche Spendenbereitschaft wecken. So beliefen sich die Spenden im Jahr 2013 anlässlich des Hochwassers in Deutschland und des Taifuns „Haiyan“ auf den Philippinen nach Erhebungen des DZI auf insgesamt 300 Millionen Euro.

Einfluss auf das Ergebnis der Umfragen hat außerdem die Formulierung der Spendenfrage bzw. die Definition der Spenden. In welcher Weise verstehen die Befragten die Frage nach Spenden? Was ist mit Spenden gemeint? Spenden an wen? Mit Ausnahme des Spendenmonitors ist allen Spendenfragen ausdrücklich 
Tabelle 6.19 Spendenfrage bzw. Spendendefinition bei den Bevölkerungsumfragen

\begin{tabular}{|c|c|}
\hline \multirow[t]{4}{*}{ Bilanz des Helfens } & $\begin{array}{l}\text { "Haben Sie im Monat [...] Spenden (freiwillige Geld-/Sachspenden) an gemein- } \\
\text { nützige (Hilfs-)Organisationen getätigt?" }\end{array}$ \\
\hline & $\begin{array}{l}\text { Wenn bejaht: Eintrag unter anderem folgender Daten im monatlichen Tage- } \\
\text { buch: Datum der Spende, Art der Spende (Geldspende oder Sachspende) und } \\
\text { Spendenbetrag. Dabei werden die Spenden wie folgt definiert: }\end{array}$ \\
\hline & $\begin{array}{l}\text { „Freiwillige Geld- und Sachspenden an gemeinnützige Organisationen/Hilfsor- } \\
\text { ganisationen/Wohltätigkeitsorganisationen. Keine Spenden an politische Par- } \\
\text { teien, politische Organisationen/keine gerichtlich veranlassten Spenden/keine } \\
\text { Lotterien, wie zum Beispiel Aktion Mensch, ARD Fernsehlotterie/keine Mitglieds- } \\
\text { beiträge." }\end{array}$ \\
\hline & $\begin{array}{l}\text { Quelle: Tagebuch Spenden des GfK Charity*Scope, Zitat mit freundlicher Geneh- } \\
\text { migung der GfK }\end{array}$ \\
\hline \multirow[t]{3}{*}{ Freiwilligensurvey } & $\begin{array}{l}\text { "Manche Menschen leisten gelegentlich oder regelmäßig Geldspenden für so- } \\
\text { ziale oder gemeinnützige Zwecke. Haben Sie in den letzten } 12 \text { Monaten solche } \\
\text { Spenden geleistet?" }\end{array}$ \\
\hline & $\begin{array}{l}\text { Wenn bejaht: Frage nach Größenordnung der Spenden (bis } 100 \text { Euro, } 101 \text { bis } \\
500 \text { Euro, } 501 \text { bis } 1000 \text { Euro, über } 1000 \text { Euro). }\end{array}$ \\
\hline & Quelle: Hameister, N., \& Vogel, C. (2017) \\
\hline \multirow[t]{3}{*}{ SOEP } & $\begin{array}{l}\text { "Wir haben nun eine Frage zu Spenden. Unter Spenden verstehen wir hier das } \\
\text { Geben von Geld für soziale, kirchliche, kulturelle, gemeinnützige und wohltäti- } \\
\text { ge Zwecke, ohne dass man dafür direkte Gegenleistungen erhält. Dies können } \\
\text { größere Beträge sein, aber auch kleinere, die man zum Beispiel in eine Sammel- } \\
\text { büchse steckt. Auch die Kollekte in der Kirche zählen wir dazu. Haben Sie im } \\
\text { letzten Jahr, also 2014, Geld gespendet - Mitgliedsbeiträge nicht mitgerech- } \\
\text { net?" }\end{array}$ \\
\hline & $\begin{array}{l}\text { Wenn bejaht: „Wie hoch war der Gesamtbetrag, den Sie im letzten Jahr gespen- } \\
\text { det haben?“ }\end{array}$ \\
\hline & Quelle: Personenfragebogen SOEP 2015 \\
\hline \multirow[t]{3}{*}{ Spendenmonitor } & $\begin{array}{l}\text { "Haben Sie innerhalb der letzten } 12 \text { Monate mindestens einmal für eine ge- } \\
\text { meinnützige Organisation gespendet?" }\end{array}$ \\
\hline & $\begin{array}{l}\text { Wenn bejaht: „Wie viel haben Sie dann insgesamt für gemeinnützige und kirch- } \\
\text { liche Zwecke (ohne Kirchensteuer) gespendet?" }\end{array}$ \\
\hline & Quelle: Deutscher Spendenmonitor 2016 \\
\hline
\end{tabular}

Quelle: Eigene Darstellung: DZI. 
zu entnehmen, dass es um Geldspenden geht. Die Erläuterungen oder Hinweise dazu fallen jedoch unterschiedlich aus. Im Freiwilligensurvey und Spendenmonitor ist die Spendenfrage jeweils knapp gehalten. Die Bilanz des Helfens und das SOEP geben nähere Hinweise. Beide schließen Mitgliedsbeiträge explizit aus. Das SOEP fokussiert sich im Übrigen eher darauf, was zu den Spenden gehört, zum Beispiel auch Spenden in Sammelbüchsen und Kollekten in Kirchen. Die Bilanz des Helfens stellt eher klar, was nicht dazu zählt, zum Beispiel Spenden an politische Parteien sowie gerichtlich veranlasste Spenden.

Eine Rolle dürfte zudem spielen, dass die Spendenfrage im SOEP auf das gesamte Spendenspektrum abzielt. Gefragt wird nach Spenden für „soziale, kirchliche, kulturelle, gemeinnützige und wohltätige Zwecke“. Der Freiwilligensurvey fragt etwas enger gefasst nach Spenden für „soziale und gemeinnützige Zwecke“. Beim Spendenmonitor und bei der Bilanz des Helfens geht es demgegenüber nur um Spenden an „gemeinnützige Organisationen“ bzw. „gemeinnützige (Hilfs-) Organisationen".

Sofern die Befragten die Spendenfrage grundsätzlich bejahen, schließen sich in den Umfragen Fragen nach der Höhe der Spenden an. Mit Ausnahme des Freiwilligensurveys wird dabei nach dem konkreten Spendenbetrag gefragt. Der Freiwilligensurvey ermittelt hingegen nur bestimmte Spendenkategorien (bis 100 Euro, 101 bis 500 Euro, 501 bis 1000 Euro, über 1000 Euro). Dies hat zur Folge, dass die Hochrechnung auf das Gesamtaufkommen beim Freiwilligensurvey im Vergleich zu den anderen Umfragen auf Annahmen bezüglich der durchschnittlichen Spendenhöhe in den verschiedenen Spendenkategorien angewiesen ist. Der Freiwilligensurvey geht hierbei nach eigenen Angaben konservativ vor und setzt die 20 Prozent-Marke der jeweiligen Kategorie als Durchschnittsspende an (in der ersten Kategorie sind dies 20 Euro und zum Beispiel in der zweiten Kategorie $100+0,2 \times 400=18$ o Euro).

Ein Aspekt, der sich bei der Hochrechnung auf das Gesamtvolumen möglicherweise ebenfalls deutlich auswirken könnte, ist die Spendenkappung. Sie wird mit sogenannten "Ausreißern“ begründet. Die Bilanz des Helfens berücksichtigt bei der Hochrechnung Spenden über 2500 Euro nicht, der Freiwilligensurvey zieht die Grenze hypothetisch bei 2 ooo Euro. Beim Spendenmonitor werden Großspenden ebenfalls bei der Hochrechnung außen vor gelassen. Eine Aussage über die konkrete Grenze lässt sich hierzu in der Veröffentlichung nicht finden. Das SOEP nimmt im Unterschied zu den anderen Hochrechnungen keine Kappung vor.

Doch Angaben zu Spenden beschränken sich nicht nur auf die Daten aus Umfragen. So werden vom DZI und vom Statistischen Bundesamt andere Quellen genutzt. 
DZI Spenden-Index (Organisationserhebung): Das DZI setzt bei seiner Erhebung im Unterschied zu den vier Bevölkerungsumfragen auf der Empfängerseite der Spenden an. Das Institut erfasst die Geldspendeneinnahmen von Spenden-SiegelOrganisationen und berechnet jährlich den DZI Spenden-Index.

Der Spenden-Index umfasst die - gemessen an den Geldspenden - 30 größten Organisationen, die das Spenden-Siegel des Instituts tragen (zum Beispiel Ärzte ohne Grenzen, Brot für die Welt und Misereor). Das Geldspendenaufkommen der Indexorganisationen liegt jährlich bei etwa 1,2 Milliarden Euro. Die Geldspenden umfassen nach der Definition des DZI die Geldspenden von privaten Personen und Unternehmen. Letztere dürften dabei einen untergeordneten Anteil ausmachen. Nicht zu den Geldspenden zählen unter anderem Mitgliedsbeiträge, Nachlässe, Geldauflagen und Zuwendungen anderer Organisationen.

Das DZI nimmt keine Hochrechnung auf das Spendenvolumen vor, sondern rechnet das Spendenvolumen aus dem SOEP (Basis ist derzeit noch das Jahr 2009: 5,3 Milliarden Euro) auf Basis der Veränderungsraten des DZI SpendenIndex jährlich fort. Um Verzerrungen durch die im Index überrepräsentierten Katastrophenspenden zu minimieren, werden dabei vom DZI außerordentliche Katastrophenspenden (insbesondere infolge großer medialer Spendenaufrufe) gesondert berücksichtigt. Grundlage hierfür sind fallbezogene Umfragen des DZI bei allen relevanten Spendenorganisationen in Deutschland (vgl. Tabelle 6.20).

Tabelle 6.20 Außerordentliche Katastrophenspenden in Deutschland (in Euro)

\begin{tabular}{lllll}
\hline Jahr & Katastrophenfall & Mio. & Katastrophenfall & Mio. \\
\hline 2002 & Hochwasser Deutschland & 350 & - & - \\
2005 & Tsunami Südostasien $(2004,2005)$ & 670 & Erdbeben Pakistan & 80 \\
2010 & Erdbeben Haiti & 230 & Flut Pakistan & 200 \\
2011 & Tsunami Japan & 71 & Hungersnot Ostafrika & 193 \\
2013 & Hochwasser Deutschland & 158 & Taifun Philippinen & 144 \\
2014 & Ebola-Epidemie Westafrika & 45 & Flüchtlingshilfe Naher Osten & 54 \\
2015 & Erdbeben Nepal & 116 & Flüchtlingshilfe In-/Ausland & 117 \\
2017 & Hungersnot Afrika & $83^{*}$ & - & - \\
\hline
\end{tabular}

* Stand Ende August 2017. Quelle: DZI Spenden-Almanach 2017. 
Lohn- und Einkommensteuerstatistik: Informationen zur Spendentätigkeit lassen sich nicht zuletzt der Lohn- und Einkommensteuerstatistik des Statistischen Bundesamtes entnehmen. Wegen der beim Besteuerungsverfahren zu berücksichtigenden Fristen liegt die jeweilige jährliche Statistik erst nach etwa dreieinhalb Jahren vor. Die jüngst verfügbare Lohn- und Einkommensteuerstatistik bezieht sich auf das Veranlagungsjahr 2013.

Die im Jahr 2013 steuerlich geltend gemachten Spenden und Mitgliedsbeiträge zur Förderung steuerbegünstigter Zwecke (ohne Spenden an Parteien und in den Vermögensstock von Stiftungen etc.) belaufen sich laut Lohn- und Einkommensteuerstatistik auf 6,8 Milliarden Euro. Mitgliedsbeiträge, denen eine Gegenleistung gegenübersteht (zum Beispiel an Sportvereine), fallen nicht hierunter. Sie sind nicht steuerbegünstigt. In den Spenden sind auch Sachspenden enthalten. Die Sachspenden und Mitgliedsbeiträge für steuerbegünstigte Zwecke dürften im Vergleich zu den Geldspenden quantitativ eine eher geringfügige Rolle spielen.

Die geltend gemachten Spenden und Mitgliedsbeiträge für steuerbegünstigte Zwecke gehen auf 9,9 Millionen Steuerpflichtige zurück, wobei zusammenveranlagte Ehepaare und Personen in eingetragenen Lebenspartnerschaften als ein Steuerpflichtiger gezählt werden.

Eine Anfrage des DZI beim Statistischen Bundesamt hat ergeben, dass in den geltend gemachten Spenden und Mitgliedsbeiträgen - dem Zweck der Lohn- und Einkommensteuerstatistik entsprechend - auch Vorträge enthalten sind. Nach Auskunft des Statistischen Bundesamtes kann es hier über die Jahre zu Doppelund Mehrfacherfassungen kommen. Vorträge entstehen, wenn geltend gemachte Spenden und Mitgliedsbeiträge in einem Jahr nicht oder nicht vollumfänglich steuerlich berücksichtigt werden können, etwa aufgrund der Höchstgrenzen bei der steuerlichen Absetzbarkeit. Die Vorträge auf das Jahr 2013 belaufen sich auskunftsgemäß auf 2,5 Milliarden Euro.

Zieht man von den geltend gemachten Spenden und Mitgliedsbeiträgen die Vorträge ab, so ergibt sich für 2013 ein Volumen von 4,3 Milliarden Euro. Dieser Betrag entspricht ungefähr der Größenordnung, die die Steuerpflichtigen gemäß einer ergänzenden Recherche des Statistischen Bundesamtes in den Einkommensteuerformularen 2013 insgesamt bei den Spenden und Mitgliedsbeiträgen für steuerbegünstigte Zwecke angegeben haben (4,9 Milliarden Euro).

Das tatsächliche Spendenaufkommen in Deutschland dürfte aus verschiedenen Gründen deutlich über diesem Betrag liegen. Denn nicht alle Spenden werden steuerlich erklärt bzw. können steuerlich erklärt werden (zum Beispiel Barspenden in Büchsen, Kollekten in Kirchen sowie Spenden für nicht als steuerbegünstigt anerkannte Zwecke). Außerdem liegen nach Auskunft des Statistischen Bundesamtes für das Jahr 2013 von 12,8 Millionen Steuerpflichtigen lediglich elektronische Lohnsteuerbescheinigungen vor. Diese Steuerpflichtigen haben keine 
Steuererklärung abgegeben. Spenden, die von ihnen geleistet wurden, sind in der Statistik ebenfalls nicht enthalten. Zudem ist ein großer Anteil der RentnerInnen aufgrund ihrer Einkommenshöhe (noch) nicht steuerpflichtig. Gerade Ältere spenden aber zu einem hohen Anteil.

\subsection{Literatur}

Braun, S., Hansen, S., \& Langner, R. (2013). Bürgerschaftliches Engagement an Schulen. Eine empirische Untersuchung über Schulfördervereine. Wiesbaden: Springer VS.

Deutsches Zentralinstitut für soziale Fragen (2017). DZI Spenden-Almanach 2017.

Eckl, M. (2015). Die Ökonomisierung der Sozialen Arbeit - Eine systemtheoretische und empirische Analyse. Journal des DGSSA, 5 (8), S. 72-88.

Emrich, Eike/Pitsch, Werner/Papathanassiou, Vassilios (2001): Die Sportvereine. Ein Versuch auf empirischer Grundlage. 1. Aufl. Schorndorf: Hofmann (Schriftenreihe des Bundesinstituts für Sportwissenschaft, 106). S. 116.

Freise, M. (2016). Substituting for the State? Friendship Societies in Germany. Voluntas, 28 (1), S. 184-203.

GfK \& Deutscher Spendenrat (2017). Bilanz des Helfens 2017. Pressemitteilungen Deutscher Spendenrat. http://www.spendenrat.de/2017/o2/28/bilanz-des-helfens2017/. Zugegriffen: 26. Oktober 2017.

Hameister, N., \& Vogel, C. (2017). Geldspenden, Spendenbeträge und freiwilliges Engagement. In J. Simonson, C. Vogel \& C. Tesch-Römer (Hrsg.), Freiwilliges Engagement in Deutschland - Der Deutsche Freiwilligensurvey 2014 (S. 217-234). Springer Link.

Horch, H.-D. (1992). Geld, Macht und Engagement in freiwilligen Vereinigungen. Grundlagen einer Wirtschaftssoziologie von Non-Profit-Organisationen. Berlin: Duncker \& Humblot.

Kantar TNS \& Deutscher Fundraising Verband (2016). Deutscher Spendenmonitor 2016. Pressemitteilungen Deutscher Fundraising Verband.

Littich, E., \& Schober, C. (2013). Finanzierung von NPOs. In R. Simsa, M. Meyer \& C. Badelt (Hrsg.), Handbuch der Nonprofit-Organisationen. Strukturen und Management (S. 289-312). s. I.: Schäffer-Poeschel Verlag für Wirtschaft Steuern Recht $\mathrm{GmbH}$.

Priemer, J. (2015). ZiviZ-Survey. Zivilgesellschaftliches Engagement für Bildung. ZiviZ im Stifterverband. Berlin.

Priemer, J., Labigne, A., \& Krimmer, H. (2016). ZiviZ-Finanzierungsstudie 2015. ZiviZ im Stifterverband. Berlin.

Priemer, J., \& Mohr, V. (2018). ZiviZ-Survey 2017. Vereine, Stiftungen und Co: Die neuen Bildungspartner? ZiviZ im Stifterverband. Berlin.

Priller, E., \& Schupp, J.(2011). Wer spendet was - und wieviel? Soziale und ökonomische Merkmale von Geld- und Blutspendern in Deutschland. In DIW Wochenbericht 29-2011. Publikationen des DIW Berlin. 
Statistisches Bundesamt (2017). Lohn- und Einkommensteuerstatistik 2013. Destatis Datenbank. Eine differenzierte Aufstellung, etwa nach Spenden und Mitgliedsbeiträgen zur Förderung steuerbegünstigter Zwecke, ist auf Anfrage beim Statistischen Bundesamt erhältlich.

Open Access Dieses Kapitel wird unter der Creative Commons Namensnennung 4.0 International Lizenz (http://creativecommons.org/licenses/by/4.0/deed.de) veröffentlicht, welche die Nutzung, Vervielfältigung, Bearbeitung, Verbreitung und Wiedergabe in jeglichem Medium und Format erlaubt, sofern Sie den/die ursprünglichen Autor(en) und die Quelle ordnungsgemäß nennen, einen Link zur Creative Commons Lizenz beifügen und angeben, ob Änderungen vorgenommen wurden.

Die in diesem Kapitel enthaltenen Bilder und sonstiges Drittmaterial unterliegen ebenfalls der genannten Creative Commons Lizenz, sofern sich aus der Abbildungslegende nichts anderes ergibt. Sofern das betreffende Material nicht unter der genannten Creative Commons Lizenz steht und die betreffende Handlung nicht nach gesetzlichen Vorschriften erlaubt ist, ist für die oben aufgeführten Weiterverwendungen des Materials die Einwilligung des jeweiligen Rechteinhabers einzuholen.

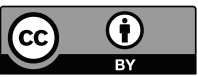

